\title{
Comprehensive analysis and identification of key genes and signaling pathways in the occurrence and metastasis of cutaneous melanoma
}

\author{
Dai Hanying ${ }^{1,2}$, Lihuang Guo ${ }^{1,2}$, Mingyue Lin ${ }^{1,2}$, Zhenbo Cheng ${ }^{1,2}$, Jiancheng $\mathbf{L i}^{1,2}{ }^{\text {, Jinxia Tang }}{ }^{1,2}$, Xisha Huan ${ }^{1,2}$, \\ Yue Huang ${ }^{1,2}$, Xu KeQian ${ }^{\text {Corresp. } 1,2}$ \\ ${ }^{1}$ Department of Laboratory Medicine, the Third Xiangya Hospital, Central South University, ChangSha, HuNan, People`s Republic of China \\ 2 Department of Laboratory Medicine, Xiangya School of Medicine, Central South University, ChangSha, HuNan, People`s Republic of China \\ Corresponding Author: Xu KeQian \\ Email address: guolihuang@csu.edu.cn
}

Background. Melanoma is a malignant tumor of melanocytes, and the incidence has increased faster than any other cancer over the past half century. Most primary melanoma can be cured by local excision, but metastatic melanoma has a poor prognosis. Cutaneous melanoma(CM) is prone to metastasis, so the research on the mechanism of melanoma occurrence and metastasis will be beneficial to diagnose early, improve treatment, and prolong life survival. In this study, we compared the gene expression of normal skin (N), primary cutaneous melanoma (PM) and metastatic cutaneous melanoma (MM) in the Gene Expression Omnibus (GEO) database. Then we identified the key genes and molecular pathways that may be involved in the development and metastasis of cutaneous melanoma, thus to discover potential markers or therapeutic targets. Methods. Three gene expression profiles (GSE7553, GSE15605 and GSE46517) were downloaded from the GEO database, which contained 225 tissue samples. R software identified the differentially expressed genes (DEGs) between pairs of N, PM and MM samples in the three sets of data. Subsequently, we analyzed the gene ontology (GO) function and Kyoto Encyclopedia of Genes and Genomes (KEGG) pathway of the DEGs, and constructed a protein-protein interaction (PPI) network. MCODE was used to seek the most important modules in PPI network, and then GO function and KEGG pathway of them were analyzed. Finally, the hub genes were calculated by the cytoHubba in Cytoscape software. The Cancer Genome Atlas (TCGA) data were analyzed using UALCAN and GEPIA to validate the hub genes and analyze the prognosis of patients. Results. 134, 317 and 147 DEGs were identified between N, PM and MM in pair. GO functions and KEGG pathways analysis results showed that the upregulated DEGs mainly concentrated in cell division, spindle microtubule, protein kinase activity and the pathway of transcriptional misregulation in cancer. The downregulated DEGs occurred in epidermis development, extracellular exosome, 
structural molecule activity, metabolic pathways and p53 signaling pathway. The PPI network obtained the most important module, whose GO function and KEGG pathway were enriched in oxidoreductase activity, cell division, cell exosomes, protein binding, structural molecule activity, and metabolic pathways. 14, 18 and 18 DEGs were identified respectively as the hub genes between $\mathrm{N}, \mathrm{PM}$ and $\mathrm{MM}$, and TCGA data confirmed the expression differences of hub genes. In addition, the overall survival curve of hub genes showed that the differences in these genes may lead to a significant decrease in overall survival of melanoma patients. Conclusions. In this study, several hub genes were found from normal skin, primary melanoma and metastatic melanoma samples. These hub genes may play an important role in the production, invasion, recurrence or death of $\mathrm{CM}$, and may provide new ideas and potential targets for its diagnosis or treatment. 


\section{Comprehensive analysis and identification of key genes and}

2 signaling pathways in the occurrence and metastasis of

\section{3 cutaneous melanoma}

4 Hanying Dai ${ }^{1,2}$, Lihuang Guo ${ }^{1,2}$, Mingyue Lin $^{1,2}$, Zhenbo Cheng ${ }^{1,2}$, Jiancheng $\mathrm{Li}^{1,2}$, Jinxia Tang ${ }^{1,2}$, Xisha

5 Huan $^{1,2}$, Yue Huang ${ }^{1,2}$, Keqian Xu Corresp, 1, 2

$6{ }^{1}$ Department of Laboratory Medicine, the Third Xiangya Hospital, Central South University, Changsha,

7 Hunan, People`s Republic of China

$8{ }^{2}$ Department of Laboratory Medicine,Xiangya School of Medicine, Central South University, Changsha,

9 Hunan, People`s Republic of China

10 Corresponding Author: Keqian Xu

11 NO.172 Tongzipo Road, Yuelu District, Changsha, Hunan, 410013, P.R. China

12 Email address: xukeqian@csu.edu.cn 


\title{
Comprehensive analysis and identification of key
} genes and signaling pathways in the occurrence and metastasis of cutaneous melanoma

\author{
Hanying Dai ${ }^{1,2}$, Lihuang Guo ${ }^{1,2}$, Mingyue Lin ${ }^{1,2}$, Zhenbo Cheng ${ }^{1,2}$, Jiancheng Li $^{1,2}$, Jinxia \\ Tang $^{1,2}$, Xisha Huan ${ }^{1,2}$, Yue Huang ${ }^{1,2}$, Keqian XuCorresp, 1, 2 \\ ${ }^{1}$ Department of Laboratory Medicine, the Third Xiangya Hospital, Central South University, \\ Changsha, Hunan, People's Republic of China \\ ${ }^{2}$ Department of Laboratory Medicine,Xiangya School of Medicine, Central South University, \\ Changsha, Hunan, People`s Republic of China
}

Corresponding Author: Keqian Xu

NO.172 Tongzipo Road, Yuelu District, Changsha, Hunan, 410013, P.R. China

Email address: xukeqian@csu.edu.cn

\section{Abstract}

Background. Melanoma is a malignant tumor of melanocytes, and the incidence has increased faster than any other cancer over the past half century. Most primary melanoma can be cured by local excision, but metastatic melanoma has a poor prognosis. Cutaneous melanoma(CM) is prone to metastasis, so the research on the mechanism of melanoma occurrence and metastasis will be beneficial to diagnose early, improve treatment, and prolong life survival. In this study, we compared the gene expression of normal skin $(\mathrm{N})$, primary cutaneous melanoma (PM) and metastatic cutaneous melanoma (MM) in the Gene Expression Omnibus (GEO) database. Then we identified the key genes and molecular pathways that may be involved in the development and metastasis of cutaneous melanoma, thus to discover potential markers or therapeutic targets. Methods. Three gene expression profiles (GSE7553, GSE15605 and GSE46517) were downloaded from the GEO database, which contained 225 tissue samples. R software identified the differentially expressed genes (DEGs) between pairs of N, PM and MM samples in the three sets of data. Subsequently, we analyzed the gene ontology (GO) function and Kyoto Encyclopedia of Genes and Genomes (KEGG) pathway of the DEGs, and constructed a proteinprotein interaction (PPI) network. MCODE was used to seek the most important modules in PPI network, and then GO function and KEGG pathway of them were analyzed. Finally, the hub genes were calculated by the cytoHubba in Cytoscape software. The Cancer Genome Atlas (TCGA) data were analyzed using UALCAN and GEPIA to validate the hub genes and analyze the prognosis of patients.

Results. 134, 317 and 147 DEGs were identified between N, PM and MM in pair. GO functions and KEGG pathways analysis results showed that the upregulated DEGs mainly concentrated in cell division, spindle microtubule, protein kinase activity and the pathway of transcriptional misregulation in cancer. The downregulated DEGs occurred in epidermis development, 
52 extracellular exosome, structural molecule activity, metabolic pathways and p53 signaling

53

54

55

56

57

58

59

60

61

62

63

64

65

66

67

68

69

70

71

72

73

74

75

76

77

78

79

80

81

82

83

84

85

86

87

88

89

90

91

pathway. The PPI network obtained the most important module, whose GO function and KEGG pathway were enriched in oxidoreductase activity, cell division, cell exosomes, protein binding, structural molecule activity, and metabolic pathways. 14, 18 and 18 DEGs were identified respectively as the hub genes between N, PM and MM, and TCGA data confirmed the expression differences of hub genes. In addition, the overall survival curve of hub genes showed that the differences in these genes may lead to a significant decrease in overall survival of melanoma patients.

Conclusions. In this study, several hub genes were found from normal skin, primary melanoma and metastatic melanoma samples. These hub genes may play an important role in the production, invasion, recurrence or death of $\mathrm{CM}$, and may provide new ideas and potential targets for its diagnosis or treatment.

\section{Introduction}

Cutaneous melanoma (CM) is the most dangerous type of skin cancer. It accounts for approximately 232,100 new cases of CM around the world each year, including 55,500 deaths ${ }^{[1]}$, and it ranks 15 th among the most common cancers in the world ${ }^{[2]}$. CM is one of the most aggressive and metastatic human cancers, and compared with other cancer types, it can spread from a small primary tumor to multiple sites throughout the body ${ }^{[3]}$. Although primary cutaneous melanoma can be removed and cured through the operation, when a few millimeters thick skin lesion is found, it represents an advanced stage, and there is a high chance of distant visceral metastasis $^{[4]}$. Once metastatic foci are established in distant organs, the 5-year overall survival rate of melanoma patients drops sharply to less than $10 \%{ }^{[5]}$. Therefore, it is urgent to identify the mechanisms that drive the occurrence and metastasis of $\mathrm{CM}$, and to develop effective therapeutic strategies. Studies had classified the somatic mutations and expression profiles of metastatic melanoma ${ }^{[6]}$, but the mechanisms of CM evolution and metastasis have not been fully understood $^{[7]}$. Understanding the gene expression changes during the development of CM will help to develop new biomarkers and therapeutic targets for the diagnosis and treatment of patients.

Gene expression microarray technology can be used to understand the biology associated with cancers, gene mutations and abnormal biological pathways, as well as to predict the diagnosis, treatment, prognosis or metastasis of patients ${ }^{[8]}$. The results of microarray technology provide a wealth of information, thus, the data stored in public databases can be reintegrated and bioinformatics analyzed to search for new clues about the pathological mechanisms of cancers through computers rather than laboratories ${ }^{[9]}$. In recent years, a large number of studies have predicted the key genes, signaling pathways and protein functions ${ }^{[10]}$ of many cancers by analyzing the patients' genetic profiles. For example, the pathogenesis and metastasis mechanism of colorectal cancer ${ }^{[11]}$, prostate cancer ${ }^{[12]}$, breast cancer ${ }^{[13]}$ and other cancers had been explored. A number of studies have done bioinformatics analysis of CM. Chen et al. ${ }^{[14]}$ compared the DEGs between normal skin and melanoma, then used bioinformatics methods to

Peer) reviewing PDF | (2020:06:49579:2:0:NEW 29 Sep 2020) 
92 analyze and identify the pathogenesis of CM. Wang et al. ${ }^{[15]}$ compared the expression of CD38

93 in the tissues of healthy people and melanoma patients in the TCGA database, and analyzed the

94 occurrence of subtypes and promoter methylation, so as to conclude that CD38 may be a

95 potential biomarker for CM. Some studies obtained differentially expressed non-coding RNAs

96 by analyzing the microRNA and lncRNA of melanoma, which proposed more possibilities for its

97 occurrence and development mechanism ${ }^{[16]}$. Meanwhile, some articles have reported the

98 metastasis of CM. For example, Chen et al. ${ }^{[17]}$ analyzed the gene expression of primary and

99 metastatic melanoma in a database and obtained some candidate genes for metastasis. Wang et

100 al. ${ }^{[18]}$ comprehensively analyzed the gene expression of PM and MM in TCGA and constructed a

101 competitive endogenous RNA (ceRNA) network, then proposed a new idea that non-coding

102 RNA and mRNA may act together on the metastasis of melanoma.

103 However, previous studies have mainly focused on the analysis of PM and N samples. Several 104 studies have also explored the metastasis of CM, but the entire progression of melanoma has not 105 been analyzed from the perspective of occurrence and development, nor has it been compared by 106 combining multiple data sets. In this study, we downloaded three gene expression profiles 107 (GSE7553, GSE15605, and GSE46517) from the GEO, which all included N, PM and MM 108 samples. DEGs among N, PM and MM were determined by gene expression profiling.

109 Subsequently, GO functions, KEGG pathways and PPI network analyses were performed on

110 DEGs. Finally, verification and survival analysis were performed on identified hub genes, which 111 may be potential biomarkers and therapeutic targets in the occurrence and transfer of $\mathrm{CM}$. The

112 flow chart is shown in Figure 1.

113

\section{Materials \& Methods}

115 GEO gene expression data

116 Three gene expression datasets (GSE7553 ${ }^{[19]}$, GSE15605 ${ }^{[20]}$, and GSE46517 $7^{[21]}$ ) were obtained

117 from the GEO database (http://www.ncbi.nlm.nih.gov/geo). The file type of the original gene

118 expression data set was CEL, and the platform file contained probe ID, gene marker and entrez

119 gene ID. GSE7553 and GSE15605 were based on the GPL570 platform (Affymetrix Human

120 Genome U133 Plus 2.0 Array) and GSE46517 was based on the GPL96 platform (Affymetrix

121 Human Genome U133A Array). There were a total of 225 tissue samples in the three data sets,

122 including 28 normal skin samples, 93 primary melanoma samples and 104 metastatic melanoma

123 samples.

124 Data processing and DEGs filtering

125 The raw CEL files were background-adjusted and standardized by the R software ${ }^{[22]}$. According to the annotation file, the probe ID was replaced with the corresponding gene symbol. If there were multiple probes for the same gene, the $\mathrm{R}$ language was used to calculate the average value for further analysis. Then the limma $\mathrm{R}$ package was used to screen the genes of each data set, when the p-value $<0.05$ and $\mid \log _{2}$ fold change (FC) $\mid>1$ were considered DEGs ${ }^{[23]}$. The

130 upregulated or downregulated DEGs lists were overlapped by Venn diagram

131 (http://bioinformatics.psb.ugent.be/webtools/Venn/), for subsequent function analysis. 
132

133

134

135

136

137

138

139

140

141

142

143

144

145

146

147

148

149

150

151

152

153

154

155

156

157

158

159

160

161

162

163

164

165

166

167

168

169

170

171

Using DAVID 6.8 database (https://david.ncifcrf.gov/home.jsp) to analyze the GO functions and KEGG pathways of integrated DEGs ${ }^{[24]}$. The GO terms and the KEGG pathways with $p<0.05$ were selected to be the enriched functions. GO functions analyses covered three domains: Biological Process, Cellular Component and Molecular Function.

\section{PPI network and the most important module analysis}

The PPI network was constructed by the STRING (https://string-db.org/) platform, an online tool used for revealing protein interactions and functional analysis ${ }^{[25]}$. In PPI network, each node represents a protein and each edge represents the action between proteins ${ }^{[26]}$. Then, the PPI network was visualized by Cytoscape software.

The most important module in PPI network was identified by means of the plug-in Molecular Complex Detection (MCODE) ${ }^{[27]}$. The criteria for selection were as follows: degree cut-off $=2$, node score cut-off $=0.2$, Max depth $=100$, and $k$-score $=2$. Subsequently, the GO functions and KEGG pathways analyses for genes in these modules were performed by DAVID, and $p<0.05$ was considered statistically significant.

\section{Hub genes selection and analysis}

Through 12 topological analysis methods, the cytoHubba of $\mathrm{R}$ software was used to sort the nodes in the PPI network. The hub genes consists of the overlapping results, which were obtained by the top 10 nodes of the Maximal Clique Centrality (MCC) analysis method and the degree of gene $\geq 10^{[28]}$. Subsequently, Pathway Commons Network Visualizer (PCViz), an open platform for exploring multidimensional cancer genome data, was used to analyze the association between hub genes and their co-expressed genes. The biological process analysis of hub genes was visualized by the Biological Networks Gene Oncology tool (BiNGO) plugin of Cytoscape ${ }^{[29]}$.

\section{Validation of hub genes and survival curve analysis}

The UALCAN website (http://ualcan.path.uab.edu/) was used to analyze the TCGA gene expression data, in order to compare the expression of hub genes in normal skin, primary melanoma and metastatic melanoma samples ${ }^{[30]}$. Then, the overall survival curve of each hub genes were analyzed by Gene Expression Profiling interactive analysis (GEPIA) (http://gepia.cancer-pku.cn/), and $\mathrm{p}<0.05$ was considered as a statistically significant difference ${ }^{[31]}$.

\section{Results}

\section{Identification of DEGs}

R software was used to compare the gene expression of samples from GSE7553, GSE15605 and GSE46517 data sets, and the DEGs of N, PM and MM were obtained in each data sets (table 1, table s1-9, available at https://doi.org/10.6084/m9.figshare.13019600.v1). Then, the overlaps of 134, 317, and 147 DEGs between PM and N, MM and N, and MM and PM are obtained from the three data sets, which were shown by Venn disgram (Fig. 2). Among them, there were 12 upregulated genes and 122 downregulated genes in the PM compared to $\mathrm{N}$ (table s10, available at https://doi.org/10.6084/m9.figshare.13019600.v1), 153 upregulated genes and 164 
172 downregulated genes between MM with $\mathrm{N}$ (table s11, available at

173 https://doi.org/10.6084/m9.figshare.13019600.v1), and MM had 79 upregulated genes and 68

174 downregulated genes compared with PM (table s12, available at

175 https://doi.org/10.6084/m9.figshare.13019600.v1).

176 GO functions and KEGG pathways enrichment analyses of DEGs

177 DAVID was used for GO functions and KEGG pathways enrichment analysis (Fig. 3-5, table 178 s13-15, available at https://doi.org/10.6084/m9.figshare.13019600.v1). GO functions analysis 179 results showed that compared with $\mathrm{N}$ samples, upregulated genes of PM were enriched in the 180 collagen catabolic process (BP), while downregulated genes were enriched in transcription from 181 RNA polymerase II promoter (BP), plasma membrane (CC) and structural molecule activity 182 (MF). The upregulated genes between $\mathrm{MM}$ and $\mathrm{N}$ samples were enriched in negative regulation 183 of neuron apoptotic process (BP), spindle microtubule (CC) and protein kinase activity (MF), 184 and the downregulated genes were enriched in epidermal development (BP), extracellular 185 exosome (CC) and structural molecule activity (MF). In MM and PM samples, the upregulated 186 genes mainly included cell division (BP), cytoplasm (CC), and protein binding (MF), and the 187 downregulated genes mainly included epidermis development (BP), extracellular exosomes 188 (CC), and structural molecule activity (MF).

189 The KEGG pathways of the overlapped DEGs were analyzed, the upregulated genes between $190 \mathrm{PM}$ and $\mathrm{N}$ were significantly enriched in the transcriptional misregulation in cancer, while the 191 downregulated genes were enriched in the metabolic pathways. In MM and N, the upregulated 192 DEGs enriched in the pathway in cancer and the transcriptional misregulation in cancer, and the 193 downregulated DEGs enriched in the arachidonic acid metabolism and steroid biosynthesis.

195 Complement and coagulation cascades was the top enriched term for upregulation genes of MM and PM, while the p53 signaling pathway was the top enriched term for downregulation genes.

\section{PPI network construction and the most meaningful module analysis}

The PPI network of DEGs was constructed using the STRING (Fig. 6) and the most important modules were obtained by Cytoscape (Fig. 7). The GO functions and KEGG pathways enrichment analysis showed that the important modules of PM and $\mathrm{N}$ were enriched in the

200 cholesterol biosynthetic process, mitochondrion, oxidoreductase activity and metabolic pathway

201 (Fig. 8A, table s16, available at https://doi.org/10.6084/m9.figshare.13019600.v1). The module

202

203 genes between $\mathrm{MM}$ and $\mathrm{N}$ were mainly enriched in cell division, extracellular exosomes, protein binding and oocyte meiosis pathway (Fig. 8B, table s17, available at

204 https://doi.org/10.6084/m9.figshare.13019600.v1). In the MM and PM, the module genes were

205 enriched in keratinocyte differentiation, cytoplasm, structural molecule activity, protein binding

206 and ismatch repair (Fig. 8C, table s18, available at

207 https://doi.org/10.6084/m9.figshare.13019600.v1).

\section{Hub gene selection and analysis}

209 According to the above criteria, 14, 18 and 18 genes among N, PM and MM were selected as the

210 hub genes in PPI network, and the details are shown in Table 2. PCViz online platform is used to

211 construct the hub genes and their co-expressed genes network (Fig. s1, available at 
212 https://doi.org/10.6084/m9.figshare.13019600.v1). The biological process analysis of hub genes

213 is shown in figure s2 (available at https://doi.org/10.6084/m9.figshare.13019600.v1).

214 Validation of hub genes and survival curve analysis

215 The transcription expression data of hub genes from 473 TCGA samples were analyzed using 216 UALCAN. Among them, 1 case was normal sample, 104 cases were PM samples, and 368 cases 217 were MM samples. We found that the expression of those hub genes in MM samples decreased 218 significantly compared with PM samples (Fig. 9). Therefore, the results of the candidate hub 219 genes identified by us are reliable.

220 We utilized the GEPIA online tool to analyze the samples data from TCGA and obtain the 221 overall survival curve of these hub genes in skin melanoma patients, so as to further study the 222 relationship between hub genes and patient survival and prognosis. As shown in figure 10, the 223 changes of IVL, FLG, SPRR1B, DSG3, KRT5, DSG1, KRT16, PKP1, KRT14 and DSC3 in

224

225

226

227

228

229

230

231

232

233

234

235

236

237

238

239

240

241

242

243

244

245

246

247

248

249

250

melanoma patients were associated with shortened overall survival, which suggested that these hub genes expression differences may be related to the progression and prognosis of cutaneous melanoma, thus can be used for predicting the deterioration and improvement of CM.

\section{Discussion}

Recently, many studies had carried out gene expression profiling and bioinformatics analysis on the molecular mechanism of CM occurrence, but the biological mechanism of its development and metastasis were still unclear. In this study, we downloaded three gene expression data sets from GEO and used a comprehensive bioinformatics method to directly compare the gene expression differences among N, PM and MM samples. 134, 317 and 147 DEGs, as well as 14, 18, and 18 hub genes were identified between PM and N, MM and N, MM and PM, respectively. Then, we used the online analysis website to verify the hub genes expression in TCGA samples and performed survival analysis on CM patients.

Through GO functions and KEGG pathways analyses of DEGs, we found that biological processes of upregulated genes mainly concentrated in cell division, spindle microtubule, protein kinase activity and the pathway of transcriptional misregulation in cancer. The downregulated gene mainly occurred in epidermis development, extracellular exosome, structural molecule activity, metabolic pathways and p53 signaling pathway. Studies have shown that the occurrence and metastasis of melanoma need to be realized through the promotion of cell mitosis and the growth of anti-aging and anti-apoptosis ${ }^{[32]}$. In addition, spindle microtubules can accelerate the proliferation and transfer of cells, and the regulation of metabolic pathways such as protein synthesis and transcriptional disorders can promote cell division ${ }^{[33]}$. The results showed that downregulated genes were associated with skin epidermal development, melanoma cells were produced in the basal layer of the epidermis and hair follicles, and epidermal keratinization could control the homeostasis of melanocytes ${ }^{[34]}$. Studies have found that most exosomes mediate the tumor process in the progression of melanoma ${ }^{[35]}$. Therefore, previous studies had confirmed our results. 
251

252

253

254

255

256

257

258

259

260

261

262

263

264

265

266

267

268

269

270

271

272

273

274

275

276

277

278

279

280

281

282

283

284

285

286

287

288

289

290

In the 14 hub genes between PM and N, it had been found that matrix metalloproteinases (MMP) can participate in the skin matrix remodeling through degrading and rebuilding the matrix components, and affect the proliferation, survival, vascularization, protease expression and migration of melanoma cells ${ }^{[36]}$. MMP-9 knockdown can reduce the migration and invasion of melanoma cells and inhibit epithelial-mesenchymal transformation (EMT), thus being considered as a promising molecule for the $\mathrm{CM}$ treatment ${ }^{[37]}$. Meanwhile, bone morphogenetic protein (BMPs) is involved in the regulation of MMPs and is an inevitable factor in the migration and invasion of melanoma cells ${ }^{[38]}$. Fibroblast growth factor receptor 3 (FGFR3) may promote melanoma growth, metastasis, and EMT behavior by influencing the phosphorylation levels of ERK, AKT, and EGFR ${ }^{[39]}$. The loss of EphB6 may have deleterious immunological effects in cancer progression, while Hafner et al. ${ }^{[40]}$ found that its expression decreased gradually in N, PM and MM. These previous studies had suggested that these hub genes may have a potential role in the development of CM.

In MM and PM, we found 18 hub genes through PPI network, all of which were downregulated genes. Several were associated with keratinocyte differentiation and epidermal development, such as loricrin (LOR), involucrin (IVL), filaggrin (FLG), small proline-rich protein1 (SPRR1), keratin (KRT) and plakophilin (PKP1), which may result in loss of epidermal function. Among them, some hub genes had been found to be related to the production and metastasis of melanoma, such as SPRR ${ }^{[41]}$ and PKP ${ }^{[42]}$. However, other genes had not been proven to be related to CM, such as LOR dysregulation was considered as an early indicator of potential malignant diseases, including oral submucosal fibrosis and leucoplasis ${ }^{[43]}$.

IVL was a specific and sensitive marker of cell differentiation, the expression of IVL in head and neck squamous cell carcinoma patients with or without lymph node metastasis was significantly different ${ }^{[44]}$. Loss-of-function mutations in FLG can lead to a decrease in epidermal filaggrin and its degradation products, and increase the sensitivity of $\mathrm{CM}^{[45]}$. In MM and PM, the downregulated amplitude of FLG $\left(\log _{2} \mathrm{FC}=-5.404\right)$ was less than the amplitude in $\mathrm{MM}$ and $\mathrm{N}$ $\left(\log _{2} \mathrm{FC}=-8.586\right)$, while there was no significant difference between PM and N. It showed that FLG may be related to CM transfer, and this association needs to be verified by subsequent experiments. Studies had shown that KRT5 and KRT14 were involved in HNSCC differentiation and apoptosis as the epithelial proliferative markers ${ }^{[46]}$. Meanwhile, immunohistochemical staining of KRT14 and KRT16 in PM and MM were mostly negative, and the positive distribution contributed to the diagnosis of poorly differentiated squamous cell carcinoma ${ }^{[47]}$. CDSN was expressed in hair follicles and keratinized epithelial cells, played an important role in intercellular adhesion, and was related to skin barrier function and epidermal defense pathway ${ }^{[48]}$. Studies had found that mutations in the CDSN gene could cause excessive keratosis of the skin, and lead to peeling skin disease and hypotrichosis simplex of the scalp ${ }^{[49]}$, therefore, down-regulation of CDSN may accelerate the development of CM by slowing down epidermal development.

In MM and PM, there were some hub genes associated with EMT. EMT was normally associated with embryogenesis and wound healing, but in tumor cells, it promoted tumor 
291 metastasis by enabling cells to leave the epithelium and acquire mesenchymal specificity[50]. This 292 process increased the aggressiveness of the tumor by the loss of the epithelial phenotype (E293 cadherin, desmosin, laminin-1) and the acquisition of the mesenchymal marker (N-cadherin) ${ }^{[51]}$. 294 Hub gene desmoglein1(DSG1) could control the role of keratinocytes, and contribute to the

295

296

297

298

299

300

301

302

303

304

305

306

307

308

309

310

311

312

313

314

315

316

317

318

319

320

321

322

323

324

325

326

327

328

329

330 page-like behavior in the development of melanoma ${ }^{[52]}$. Furthermore, desmocollin1(DSC1) and desmocollin3(DSC3) are members of the E-cadherin superfamily, involved in cell-cell adhesion and cell-extracellular matrix interaction. Benign melanocytes expressed high levels of Ecadherin, and during the transition to melanoma cells, E-cadherin was down-regulated and Ncadherin was up-regulated ${ }^{[33]}$. Studies had shown that desmoplakin (DSP) was a desmosomal protein involved in cell-cell adhesion. Desmosome formation was characteristic of cell differentiation and intercellular adhesion, and the loss of desmosome might accelerate the occurrence and early migration of tumor cells ${ }^{[54]}$.

There are also some hub genes between MM and PM had been found to be related to CM, for example, the down-regulation of cystatin A (CSTA) expression has become an important feature to distinguish N, PM and $\mathrm{MM}^{[55]}$. Several S100 family genes had been found to be highly expressed in PM, but low level in $\mathrm{MM}^{[56]}$. In particular, the loss of S100A7 is highly correlated with the metastasis progression score ${ }^{[57]}$. Lentin et al. ${ }^{[58]}$ observed that the anti-invasion effect of transglutaminase(TGM) might lead to the post-translational modification of some components of the cell basal membrane, thereby interfering with the metastasis of melanoma cells. The activity of TGM2 had a protective effect on the progression of melanoma in vivo ${ }^{[59]}$, but no studies had been conducted to prove the relationship between TGM1 and CM, so TGM1 may be a new potential marker.

We found that most of the above hub genes had been reported to be closely related to the generation and metastasis of $\mathrm{CM}$. Moreover, through prognostic analysis, most hub gene expression differences in CM patients were connected with overall survival, which proved the reliability of our study. There are still a few genes that had not been reported or experimentally confirmed to be associated with $\mathrm{CM}$, but some of them are related to the occurrence and development of other cancers. So they might be potential biomarkers of CM, and a large number of experiments are needed to confirm. Most hub genes, such as LOR, IVL, FLG, DSG3, TGM1, KRT16, SPRR1A, KRT14, DSP and CSTA, showed no difference in the expression of PM and $\mathrm{N}$, but significantly decreased in MM and PM, suggesting that these genes might be potential predictors of CM metastasis. The expression of some genes, such as CDSN, DSG1, DSC3, DSC1 and DSP, was downregulated in all three groups, which might be relate with the occurrence and progression of CM. It is worth noting that three genes, SPRR1B, PKP1 and S100A7, were upregulated in PM and N, but downregulated in MM and PM, which were likely to be used as novel markers to distinguish whether $\mathrm{CM}$ was metastatic or not. These assumptions need to be tested experimentally.

Compared with previous studies, we used more samples, and compared N, PM and MM in pairs, then took the intersection, so as to make the experimental results more reliable. Besides using GEO data sets, we also used TCGA data for verification, which increased the sample size 
331

332

333

334

335

336

337

338

339

340

341

342

343

344

345

346

347

348

349

350

351

352

353

354

355

356

357

358

359

360

361

362

363

364

365

366

367

368

369

370

and accuracy. However, the limitation of this study is the lack of experimental verification of hub genes. Therefore, to understand whether the hub genes are really closely related to the generation and metastasis of $\mathrm{CM}$, a large number of subsequent experiments are needed to explore.

\section{Conclusions}

In conclusion, through bioinformatics analysis, we obtained some potential key genes and biological pathways related to the occurrence and metastasis of $\mathrm{CM}$, providing directions for future research. In addition to the genes that have been reported and demonstrated, other identified key genes may be potential prognostic markers and therapeutic targets for $\mathrm{CM}$ occurrence and metastasis.

\section{Acknowledgements}

We would like to thank all teachers in Department of Laboratory Medicine for their thoughtful kindness. The present study was supported by the National Natural Science Funding of China (No.81273002 and No.81471499) and Hunan Provincial Natural Science Foundation of China (No.2019JJ40347).

\section{References}

[1] Schadendorf D, van Akkooi ACJ, Berking C, Griewank KG, Gutzmer R, Hauschild A, Stang A, Roesch A, Ugurel S. 2018. Melanoma. Lancet, 392(10151):971-984. Doi: 10.1016/S01406736(18)31559-9.

[2] Leonardi GC, Falzone L, Salemi R, Zanghì A, Spandidos DA, Mccubrey JA, Candido S, Libra M. 2018. Cutaneous melanoma: From pathogenesis to therapy (Review). Int J Oncol, 52(4):1071-1080. Doi: 10.3892/ijo.2018.4287.

[3] Bostel T, Förster R, Schlampp I, Wolf R, Serras AF, Mayer A, Bruckner T, Welzel T, Schmidberger H, Debus J, Rief H. 2016. Stability, prognostic factors and survival of spinal bone metastases in malignant melanoma patients after palliative radiotherapy. Tumori, 102(2):156-61. Doi: 10.5301/tj.5000382.

[4] Wei D. 2017. A multigene support vector machine predictor for metastasis of cutaneous melanoma. Mol Med Rep, 17(2):2907-2914. Doi: 10.3892/mmr.2017. 8219.

[5] Braeuer RR, Watson IR, Wu CJ, Mobley AK, Kamiya T, Shoshan E, Bar-Eli M. 2014. Why is melanoma so metastatic? Pigment Cell Melanoma Res, 27(1):19-36. Doi: $10.1111 / \mathrm{pcmr} .12172$.

[6] Cancer Genome Atlas Network. 2015. Genomic classification of cutaneous melanoma. Cell, 161(7):1681-96. Doi: 10.1016/j.cell.2015.05.044.

[7] Shain AH, Joseph NM, Yu R, Benhamida J, Liu S, Prow T, Ruben B, North J, Pincus L, Yeh I, Judson R, Bastian BC. 2018. Genomic and Transcriptomic Analysis Reveals Incremental Disruption of Key Signaling Pathways during Melanoma Evolution. Cancer Cel, 34(1):4555.e4. Doi: 10.1016/j.ccell.2018.06.005. 
371 [8] Tao Z, Shi A, Li R, Wang Y, Wang X, Zhao J. 2017. Microarray bioinformatics in cancer- a

372

373

374

375

376

377

378

379

380

381

382

383

384

385

386

387

388

389

390

391

392

393

394

395

396

397

398

399

400

401

402

403

404

405

406

407

408

409 review. J BUON, 22(4):838-843.

[9] Wei CY, Zhu MX, Lu NH, Peng R, Yang X, Zhang PF, Wang L, Gu JY. 2019. Bioinformatics-based analysis reveals elevated MFSD12 as a key promoter of cell proliferation and a potential therapeutic target in melanoma. Oncogene, 38(11):1876-1891. Doi: 10.1038/s41388-018-0531-6.

[10] Le NQK, Yapp EKY, Nagasundaram N, Chua MCH, Yeh HY. 2019. Computational identification of vesicular transport proteins from sequences using deep gated recurrent units architecture. Comput Struct Biotechnol J, 17:1245-1254. Doi: 10.1016/j.csbj.2019.09.005.

[11] Huang D, Sun W, Zhou Y, Li P, Chen F, Chen H, Xia D, Xu E, Lai M, Wu Y, Zhang H. 2018. Mutations of key driver genes in colorectal cancer progression and metastasis. Cancer Metastasis Rev, 37(1):173-187. Doi: 10.1007/s10555-017-9726-5.

[12] Wang Y, Guo W, Xu H, Zhu X, Yu T, Jiang Z, Jiang Q, Gang X. 2018. An extensive study of the mechanism of prostate cancer metastasis. Neoplasma, 65(2):253-261. Doi: 10.4149/neo_2018_161217N648.

[13] Bertucci F, Ng CKY, Patsouris A, Droin N, Piscuoglio S, Carbuccia N, Soria JC, Dien AT, Adnani Y, Kamal M, Garnier S, Meurice G, Jimenez M, Dogan S, Verret B, Chaffanet M, Bachelot T, Campone M, Lefeuvre C, Bonnefoi H, Dalenc F, Jacquet A, De Filippo MR, Babbar N, Birnbaum D, Filleron T, Le Tourneau C, André F. 2019. Genomic characterization of metastatic breast cancers. Nature, 569(7757): 560-564. Doi: 10.1038/s41586-019-1056-z.

[14] Chen J, Sun W, Mo N, Chen X, Yang L, Tu S, Zhang S, Liu J. 2020. Identification of key genes involved in the pathogenesis of cutaneous melanoma using bioinformatics analysis. J Int Med Res, 48(1): 300060519895867. Doi: 10.1177/0300060519895867.

[15] Wang X, Wang P, Ge L, Wang J, Naqvi SMAS, Hu S. 2020. Identification of CD38 as a potential biomarker in skin cutaneous melanoma using bioinformatics analysis. Oncol Lett, 20(4):12. Doi: 10.3892/ol.2020.11873.

[16] Li Q, Zhang LY, Wu S, Huang C, Liu J, Wang P, Cao Y. 2019. Bioinformatics Analysis Identifies MicroRNAs and Target Genes Associated with Prognosis in Patients with Melanoma. Med Sci Monit, 25:7784-7794. Doi: 10.12659/MSM.917082.

[17] Chen J, Wu F, Shi Y, Yang D, Xu M, Lai Y, Liu Y. 2019. Identification of key candidate genes involved in melanoma metastasis. Mol Med Rep, 20(2):903-914. Doi: 10.3892/mmr.2019.10314.

[18] Wang LX, Wan C, Dong ZB, Wang BH, Liu HY, Li Y. 2019. Integrative Analysis of Long Noncoding RNA (lncRNA), microRNA (miRNA) and mRNA Expression and Construction of a Competing Endogenous RNA (ceRNA) Network in Metastatic Melanoma. Med Sci Monit, 25:2896-2907. Doi: 10.12659/MSM.913881.

[19] Riker AI, Enkemann SA, Fodstad O, Liu S, Ren S, Morris C, Xi Y, Howell P, Metge B, Samant RS, Shevde LA, Li W, Eschrich S, Daud A, Ju J, Matta J. 2008. The gene expression 
410

411

412

413

414

415

416

417

418

419

420

421

422

423

424

425

426

427

428

429

430

431

432

433

434

435

436

437

438

439

440

441

442

443

444

445

446

447

448

profiles of primary and metastatic melanoma yields a transition point of tumor progression and metastasis. BMC Med Genomics, 1:13. Doi: 10.1186/1755-8794-1-13.

[20] Raskin L, Fullen DR, Giordano TJ, Thomas DG, Frohm ML, Cha KB, Ahn J, Mukherjee B, Johnson TM, Gruber SB. 2013. Transcriptome profiling identifies HMGA2 as a biomarker of melanoma progression and prognosis. J Invest Dermatol, 133(11):2585-2592. Doi: 10.1038/jid.2013.197.

[21] Kabbarah O, Nogueira C, Feng B, Nazarian RM, Bosenberg M, Wu M, Scott KL, Kwong LN, Xiao Y, Cordon-Cardo C, Granter SR, Ramaswamy S, Golub T, Duncan LM, Wagner SN, Brennan C, Chin L. 2010. Integrative genome comparison of primary and metastatic melanomas. PLoS One, 5(5):e10770. Doi: 10.1371/journal.pone.0010770.

[22] Smyth GK. 2004. Linear models and empirical bayes methods for assessing differential expression in microarray experiments. Stat Appl Genet Mol Biol, 3:Article3. Doi: 10.2202/1544-6115.1027.

[23] Zhang YJ, Sun YZ, Gao XH, Qi RQ. 2019. Integrated bioinformatic analysis of differentially expressed genes and signaling pathways in plaque psoriasis. Mol Med Rep, 20(1):225-235. Doi: 10.3892/mmr.2019.10241.

[24] Xing Z, Chu C, Chen L, Kong X. 2016. The use of Gene Ontology terms and KEGG pathways for analysis and prediction of oncogenes. Biochim Biophys Acta, 1860:2725-34. Doi: 10.1016/j.bbagen.2016.01.012.

[25] Szklarczyk D, Morris JH, Cook H, Kuhn M, Wyder S, Simonovic M, Santos A, Doncheva NT, Roth A, Bork P, Jensen LJ, von Mering C. 2017. The STRING database in 2017: quality-controlled protein-protein association networks, made broadly accessible. Nucleic Acids Res, 45:D362-D368. Doi: 10.1093/nar/gkw937.

[26] Khunlertgit N, Yoon BJ. 2016. Incorporating topological information for predicting robust cancer subnetwork markers in human protein-protein interaction network. BMC Bioinforma, 17:351. Doi: 10.1186/s12859-016-1224-1.

[27] Li L, Lei Q, Zhang S, Kong L, Qin B. 2017. Screening and identification of key biomarkers in hepatocellular carcinoma: Evidence from bioinformatic analysis. Oncol Rep, 38(5):26072618. Doi: 10.3892/or.2017.5946.

[28] Chin CH, Chen SH, Wu HH, Ho CW, Ko MT, Lin CY. 2014. cytoHubba: Identifying hub objects and sub-networks from complex interactome. BMC Syst Biol, 8(Suppl 4):S11. Doi: 10.1186/1752-0509-8-S4-S11.

[29] Maere S, Heymans K, Kuiper M. 2005. BiNGO: A Cytoscape plugin to assess overrepresentation of gene ontology categories in biological networks. Bioinformatics, 21:3448-3449. Doi: 10.1093/bioinformatics/bti551.

[30] Chandrashekar DS, Bashel B, Balasubramanya SAH, Creighton CJ, Ponce-Rodriguez I, Chakravarthi BVSK, Varambally S. 2017. UALCAN: a portal for facilitating tumor subgroup gene expression and survival analyses. Neoplasia(New York, N.Y.), 19(8):649658. Doi: 10.1016/j.neo.2017.05.002. 
449 [31] Tang Z, Li C, Kang B, Gao G, Li C, Zhang Z. 2017. GEPIA: a web server for cancer and

450

451

452

453

454

455

456

457

458

459

460

461

462

463

464

465

466

467

468

469

470

471

472

473

474

475

476

477

478

479

480

481

482

483

484

485

486

487

488 normal gene expression profiling and interactive analyses. Nucleic acids research, 45(w1):w98-w102. Doi: 10.1093/nar/gkx247.

[32] Bennett DC. 2016. Genetics of melanoma progression: the rise and fall of cell senescence. Pigment Cell Melanoma Res, 29(2):122-40. Doi: 10.1111/pcmr.12422.

[33] Soltani MH, Pichardo R, Song Z, Sangha N, Camacho F, Satyamoorthy K, Sangueza OP, Setaluri V. 2005. Microtubule-associated protein 2, a marker of neuronal differentiation, induces mitotic defects, inhibits growth of melanoma cells, and predicts metastatic potential of cutaneous melanoma. Am J Pathol, 166(6):1841-50. Dio: 10.1016/S00029440(10)62493-5.

[34] Orgaz JL, Sanz-Moreno V. 2013. Emerging molecular targets in melanoma invasion and metastasis. Pigment Cell Melanoma Res, 26(1):39-57. Doi: 10.1111/pcmr.12041.

[35] Mannavola F, D'Oronzo S, Cives M, Stucci LS, Ranieri G, Silvestris F, Tucci M. 2019. Extracellular Vesicles and Epigenetic Modifications Are Hallmarks of Melanoma Progression. Int J Mol Sci, 21(1):52. Doi: 10.3390/ijms21010052.

[36] Napoli S, Scuderi C, Gattuso G, Bella VD, Candido S, Basile MS, Libra M, Falzone L. 2020. Functional Roles of Matrix Metalloproteinases and Their Inhibitors in Melanoma. Cells, 9(5). Doi: 10.3390/cells9051151.

[37] Tian Y, Guo Y, Zhu P, Zhang D, Liu S, Tang M, Wang Y, Jin Z, Li D, Yan D, Li G, Zhu X. 2019. TRIM59 loss in M2 macrophages promotes melanoma migration and invasion by upregulating MMP-9 and Madcam1. Aging (Albany NY), 11(19):8623-8641. Doi: 10.18632/aging.102351

[38] Rothhammer T, S Braig and AK Bosserhoff. 2008. Bone morphogenetic proteins induce expression of metalloproteinases in melanoma cells and fibroblasts. Eur J Cancer, 44(16):2526-34. Doi: 10.1016/j.ejca.2008.07.029.

[39] Li L, Zhang S, Li H, Chou H. 2019. FGFR3 promotes the growth and malignancy of melanoma by influencing EMT and the phosphorylation of ERK, AKT, and EGFR. BMC Cancer, 19(1): 963. oi: 10.1186/s12885-019-6161-8.

[40] Hafner C, Bataille F, Meyer S, Becker B, Roesch A, Landthaler M, Vogt T. 2003. Loss of EphB6 expression in metastatic melanoma. Int J Oncol, 23(6):1553-9. Doi: 10.3892/ijo.23.6.1553.

[41] Koizumi H, Kartasova T, Tanaka H, Ohkawara A, Kuroki T. 1996. Differentiationassociated localization of small proline-rich protein in normal and diseased human skin. $\mathrm{Br}$ J Dermatol, 134(4):686-92. Doi: 10.1111/j.1365-2133.1996.tb06971.x.

[42] Wang HZ, Wang F, Chen PF, Zhang M, Yu MX, Wang HL, Zhao Q, Liu J. 2019. Coexpression network analysis identified that plakophilin 1 is associated with the metastasis in human melanoma. Biomed Pharmacother, 111:1234-1242. Doi: 10.1016/j.biopha.2018.12.135.

[43] S N, Joshua E, K R, Thavarajah R, Rao UK. 2019. Loricrin expression and its implication in oral submucous fibrosis, hyperkeratosis and normal mucosa with association to habits - An 
489

490

491

492

493

494

495

496

497

498

499

500

501

502

503

504

505

506

507

508

509

510

511

512

513

514

515

516

517

518

519

520

521

522

523

524

525

526

527

528

immunohistochemical study. J Oral Biol Craniofac Res, 9(3):226-231. Doi:

10.1016/j.jobcr.2019.05.004.

[44] Jin Y, Qin X. 2020. Co-expression network-based identification of biomarkers correlated with the lymph node metastasis of patients with head and neck squamous cell carcinoma. Biosci Rep, 40(2): BSR20194067. Doi: 10.1042/BSR20194067.

[45] Thyssen JP, Andersen YMF, Balslev E, Szecsi PB, Stender S, Kaae J, Linneberg A, Skov L. 2018. Loss-of-function mutations in filaggrin gene and malignant melanoma: a case-control study. J Eur Acad Dermatol Venereol, 32(2):242-244. Doi: 10.1111/jdv.14532.

[46] Wang L, Feng Z, Wu H, Zhang S, Pu Y, Bian H, Wang Y, Guo C. 2014. Melanoma differentiation-associated gene-7/interleukin-24 as a potential prognostic biomarker and second primary malignancy indicator in head and neck squamous cell carcinoma patients. Tumour Biol, 35(11):10977-85. Doi: 10.1007/s13277-014-2392-0.

[47] Safadi RA, Bader DH, Abdullah NI, Sughayer MA. 2016. Immunohistochemical expression of keratins $6,7,8,14,16,18,19$, and MNF-116 pancytokeratin in primary and metastatic melanoma of the head and neck. Oral Surg Oral Med Oral Pathol Oral Radiol, 121(5):5109. Doi: 10.1016/j.oooo.2015.11.016.

[48] Mondon P, Ringenbach C, Doridot E, Genet V. 2017. Reinforcement of barrier function and scalp homeostasis by Senkyunolide A to fight against dandruff. Int J Cosmet Sci, 39(6):617621. Doi: $10.1111 /$ ics.12417.

[49] van der Velden JJAJ, van Geel M, Engelhart JJ, Jonkman MF, Steijlen PM. 2020. Mutations in the CDSN gene cause peeling skin disease and hypotrichosis simplex of the scalp. $J$ Dermatol, 47(1):3-7. Doi: 10.1111/1346-8138.15136.

[50] Hodorogea A, Calinescu A, Antohe M, Balaban M, Nedelcu RI, Turcu G, Ion DA, Badarau IA, Popescu CM, Popescu R, Popp C, Cioplea M, Nichita L, Hulea I, Brinzea A. 2019. Epithelial-Mesenchymal Transition in Skin Cancers: A Review. Anal Cell Pathol (Amst), 2019:3851576. Doi: 10.1155/2019/3851576. eCollection 2019.

[51] Kalluri R, Robert A. 2009. The basics of epithelial-mesenchymal transition. J Clin Invest, 119(6):1420-1428. Doi: 10.1172/JCI39104.

[52] Arnette CR, Roth-Carter QR, Koetsier JL, Broussard JA, Burks HE, Cheng K, Amadi C, Gerami P, Johnson JL, Green KJ. 2020. Keratinocyte cadherin desmoglein 1 controls melanocyte behavior through paracrine signaling. Pigment Cell Melanoma Res, 33(2):305317. Doi: 10.1111/pcmr.12826.

[53] Jaeger J, Koczan D, Thiesen HJ, Ibrahim SM, Gross G, Spang R, Kunz M. 2007. Gene expression signatures for tumor progression, tumor subtype, and tumor thickness in lasermicrodissected melanoma tissues. Clin Cancer Res, 1;13(3):806-15. Doi: 10.1158/10780432.CCR-06-1820.

[54] Walia V, Prickett TD, Kim JS, Gartner JJ, Lin JC, Zhou M, Rosenberg SA, Elble RC, Solomon DA, Waldman T, Samuels Y. 2014. Mutational and functional analysis of the tumor-suppressor PTPRD in human melanoma. Hum Mutat, 35(11):1301-10. Doi: 10.1002/humu.22630. 
529 [55] Wallin H, Apelqvist J, Andersson F, Ekström U, Abrahamson M. 2017. Low-level

530

531

532

533

534

535

536

537

538

539

540

541

542

543

544

545 internalization of cystatin $\mathrm{E} / \mathrm{M}$ affects legumain activity and migration of melanoma cells. J Biol Chem, 292(35):14413-14424. Doi: 10.1074/jbc.M117.776138.

[56] Xiong TF, Pan FQ, Li D. 2019. Expression and clinical significance of S100 family genes in patients with melanoma. Melanoma Res, 29(1):23-29. Doi: 10.1097/CMR.0000000000000512.

[57] Bhalla S, Kaur H, Dhall A, Raghava GPS. 2019. Prediction and Analysis of Skin Cancer Progression using Genomics Profiles of Patients. Sci Rep, 9(1):15790. Doi: 10.1038/s41598-019-52134-4.

[58] Lentini A, Provenzano B, Caraglia M, Shevchenko A, Abbruzzese A, Beninati S. 2008. Impairment of the metastatic activity of melanoma cells by transglutaminase-catalyzed incorporation of polyamines into laminin and Matrigel. Amino Acids, 34(2):251-6. Doi: 10.1007/s00726-007-0505-4.

[59] Facchiano F, D'Arcangelo D, Lentini A, Rossi S, Senatore C, Pannellini T, Tabolacci C, Facchiano AM, Facchiano A, Beninati S. 2013. Tissue transglutaminase activity protects from cutaneous melanoma metastatic dissemination: an in vivo study. Amino Acids, 44(1):53-61. Doi: 10.1007/s00726-012-1351-6. 


\section{Table 1 (on next page)}

The information of GEO dataset and DEGs. 


\begin{tabular}{|c|c|c|c|c|c|c|c|}
\hline \multirow{2}{*}{$\begin{array}{c}\text { GEO } \\
\text { datasets }\end{array}$} & \multirow{2}{*}{ Platform } & \multirow{2}{*}{$\begin{array}{l}\text { Normal } \\
\text { skin(N) }\end{array}$} & \multirow{2}{*}{$\begin{array}{l}\text { Primary } \\
\text { melanoma } \\
\quad(\mathrm{PM})\end{array}$} & \multirow{2}{*}{$\begin{array}{l}\text { Metastatic } \\
\text { melanoma } \\
\quad(\mathrm{MM})\end{array}$} & \multicolumn{3}{|c|}{ Differentially expressed genes(DEGs) } \\
\hline & & & & & $\mathrm{PM}$ and $\mathrm{N}$ & $\mathrm{MM}$ and $\mathrm{N}$ & $\mathrm{MM}$ and $\mathrm{PM}$ \\
\hline GSE7553 & GPL570 & 4 & 16 & 40 & 843 & 1129 & 929 \\
\hline GSE15605 & GPL570 & 16 & 46 & 12 & 2605 & 2829 & 1560 \\
\hline GSE46517 & GPL96 & 8 & 31 & 52 & 668 & 813 & 2466 \\
\hline
\end{tabular}

1 
Table 2 (on next page)

The hub genes among normal skin, primary cutaneous melanoma and metastatic cutaneous melanoma samples. 


\begin{tabular}{llll}
\hline & PM and N & MM and N & MM and PM \\
\hline Upregulated & MMP9, MMP1 & AURKA, CCNB1, & \\
& & TPX2 & \\
Downregulated & KRT19, BMP2, & LOR, FLG, JUP, & LOR, IVL, FLG, \\
& HMGCS2, KLF4, & DSC1, SPRR1B, & SPRR1B, DSG3, \\
& EPHB3, EFNA3, PIP, & DSG1, DSP, SPRR1A, & KRT5, CDSN, TGM1, \\
& ADH1B, FGFR3, & BUB1B, KRT5, & DSG1, KRT16, \\
& SDC1, CEBPA, & KRT14, CDSN, IVL, & SPRR1A, PKP1, \\
& EPHB6 & DSG3, TGM1 & KRT14, DSC3, DSP, \\
& & CSTA, S100A7, DSC1 \\
\hline
\end{tabular}

1 
Figure 1

Experimental flow chart.

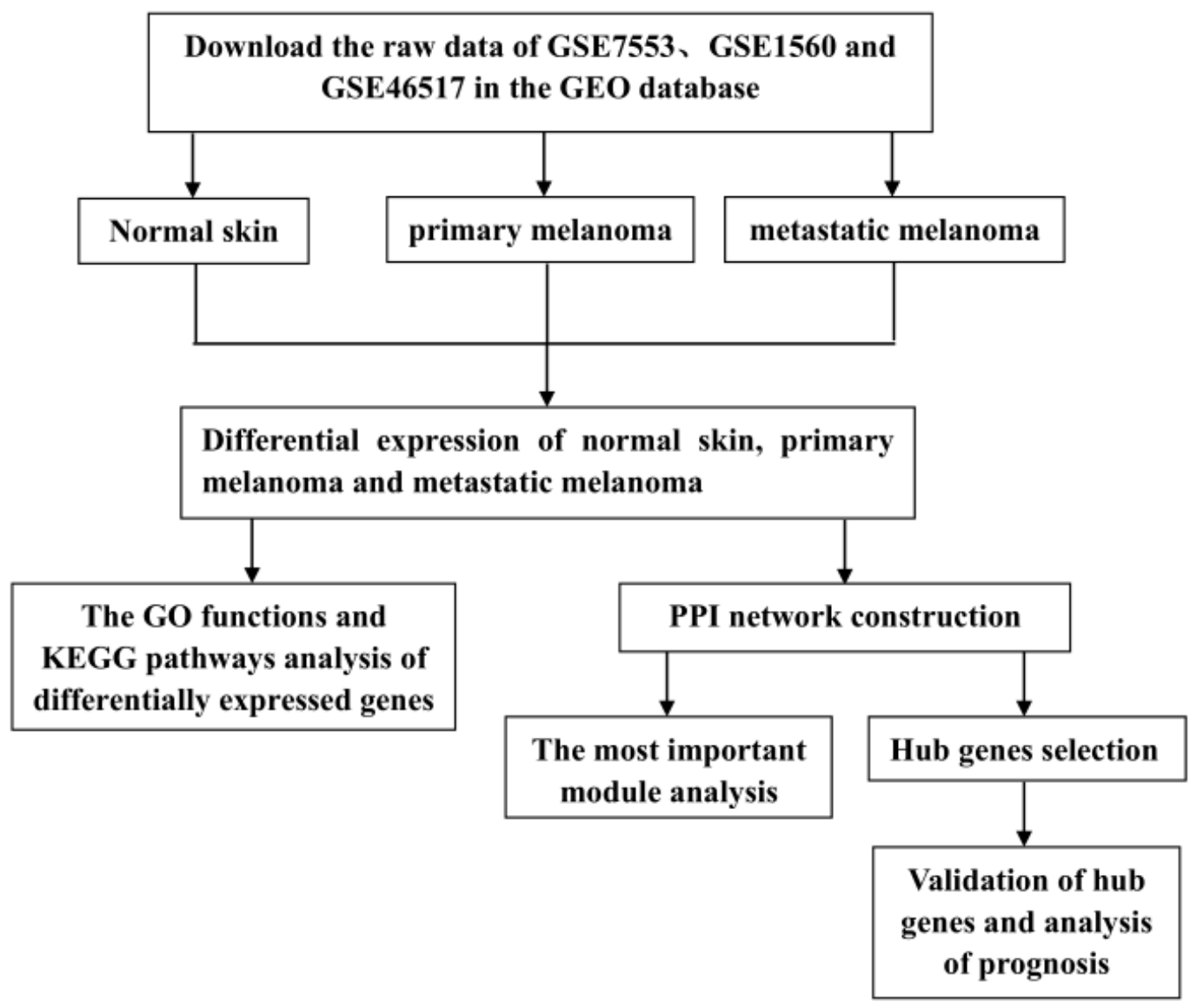




\section{Figure 2}

Venn diagram of DEGs in GSE7553, GSE15605 and GSE46517.

According to $\mid \log _{2}$ fold change $(F C) \mid>1$ and $p$-value $<0.05$, DEGs were identified along normal skin, primary cutaneous melanoma and metastatic cutaneous melanoma samples in GSE7553, GSE15605 and GSE46517 data sets. (A) The overlap of 134 DEGs was shown between the PM and N samples of the three data sets. (B) The overlap of 317 DEGs was shown between MM and $\mathrm{N}$ of the three data sets. (C) The overlap of 147 DEGs was shown between the MM and PM of the three data sets.

A

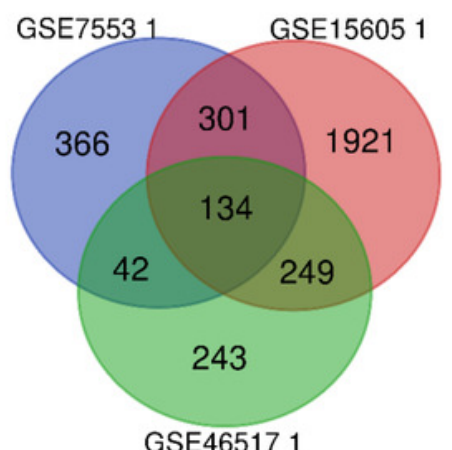

B

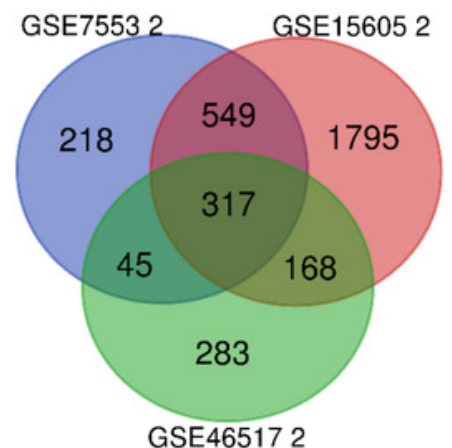

C

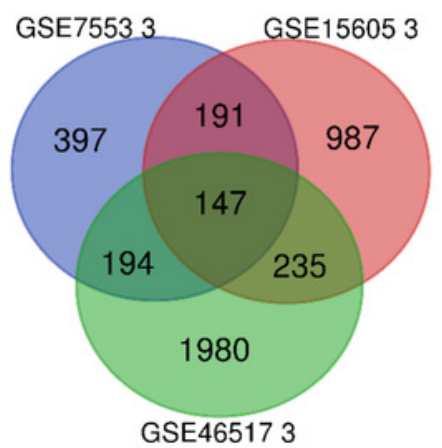




\section{Figure 3}

GO functions and KEGG pathways enrichment analysis of the upregulated and downregulated genes between primary cutaneous melanoma and normal skin.

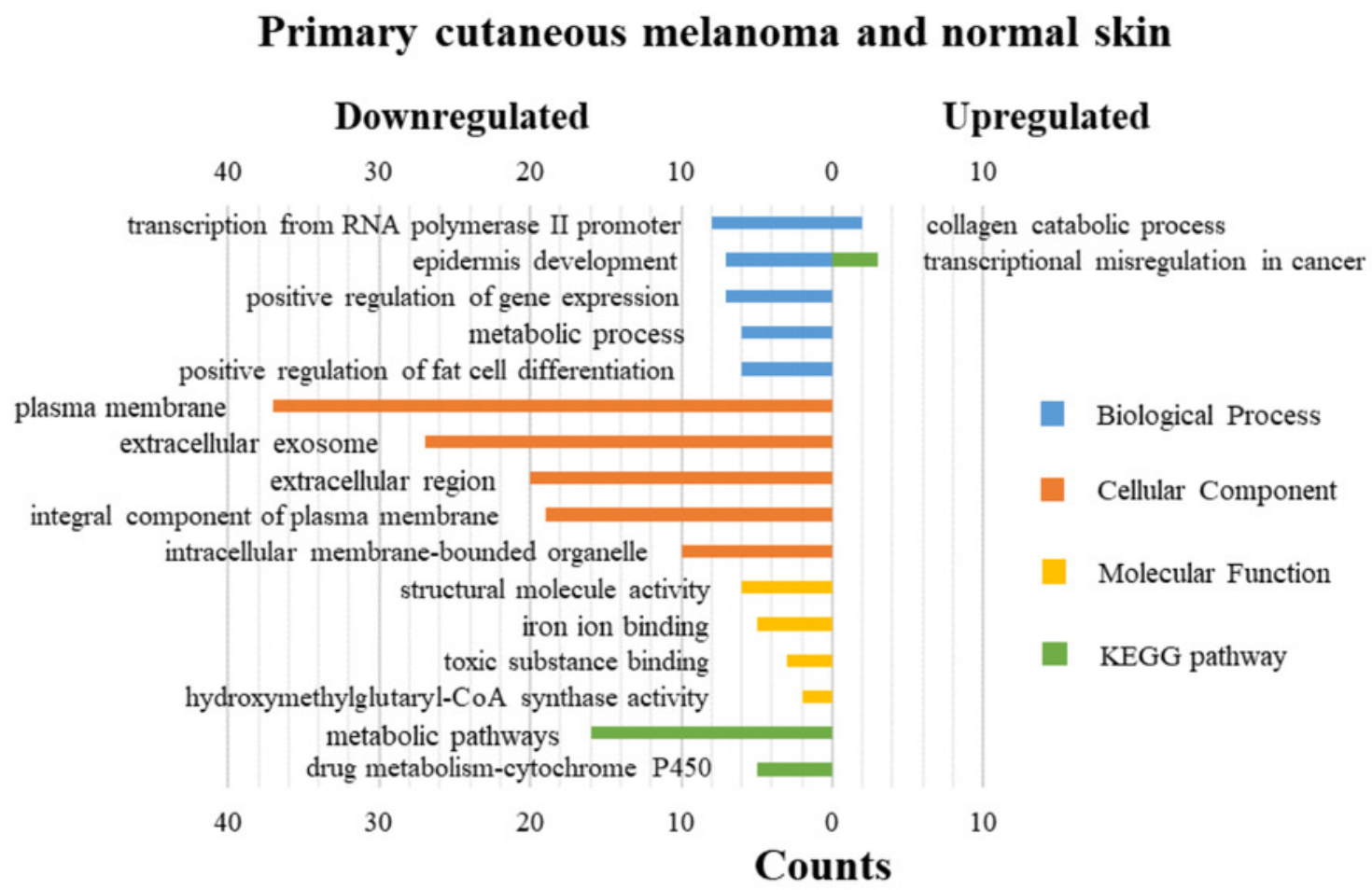




\section{Figure 4}

\section{GO functions and KEGG pathways enrichment analysis of the upregulated and downregulated genes between metastatic cutaneous melanoma and normal skin.}

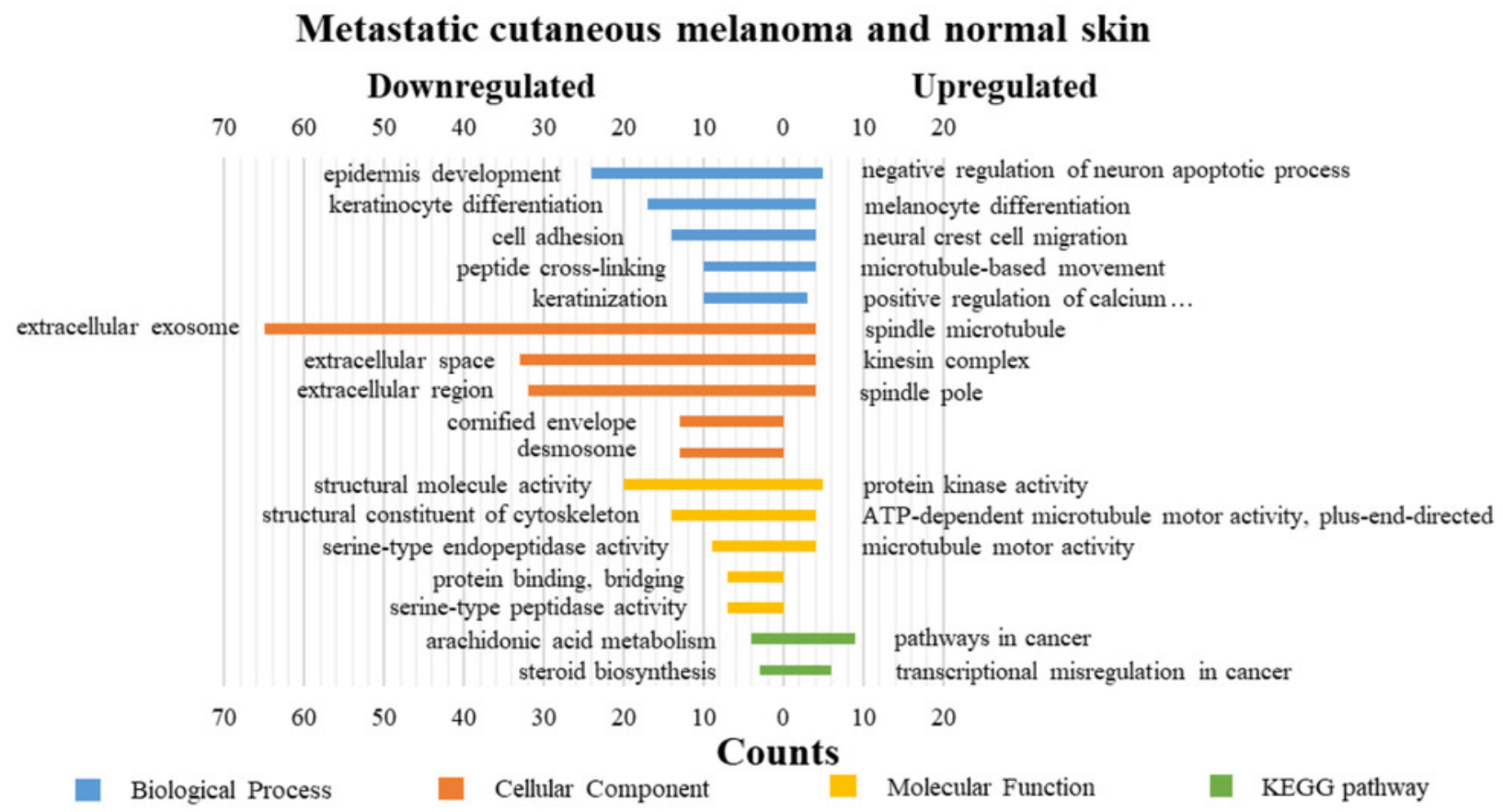


Figure 5

GO functions and KEGG pathways enrichment analysis of the upregulated and downregulated genes between metastatic cutaneous melanoma and primary cutaneous melanoma.

\section{Metastatic cutaneous melanoma and primary cutaneous melanoma}

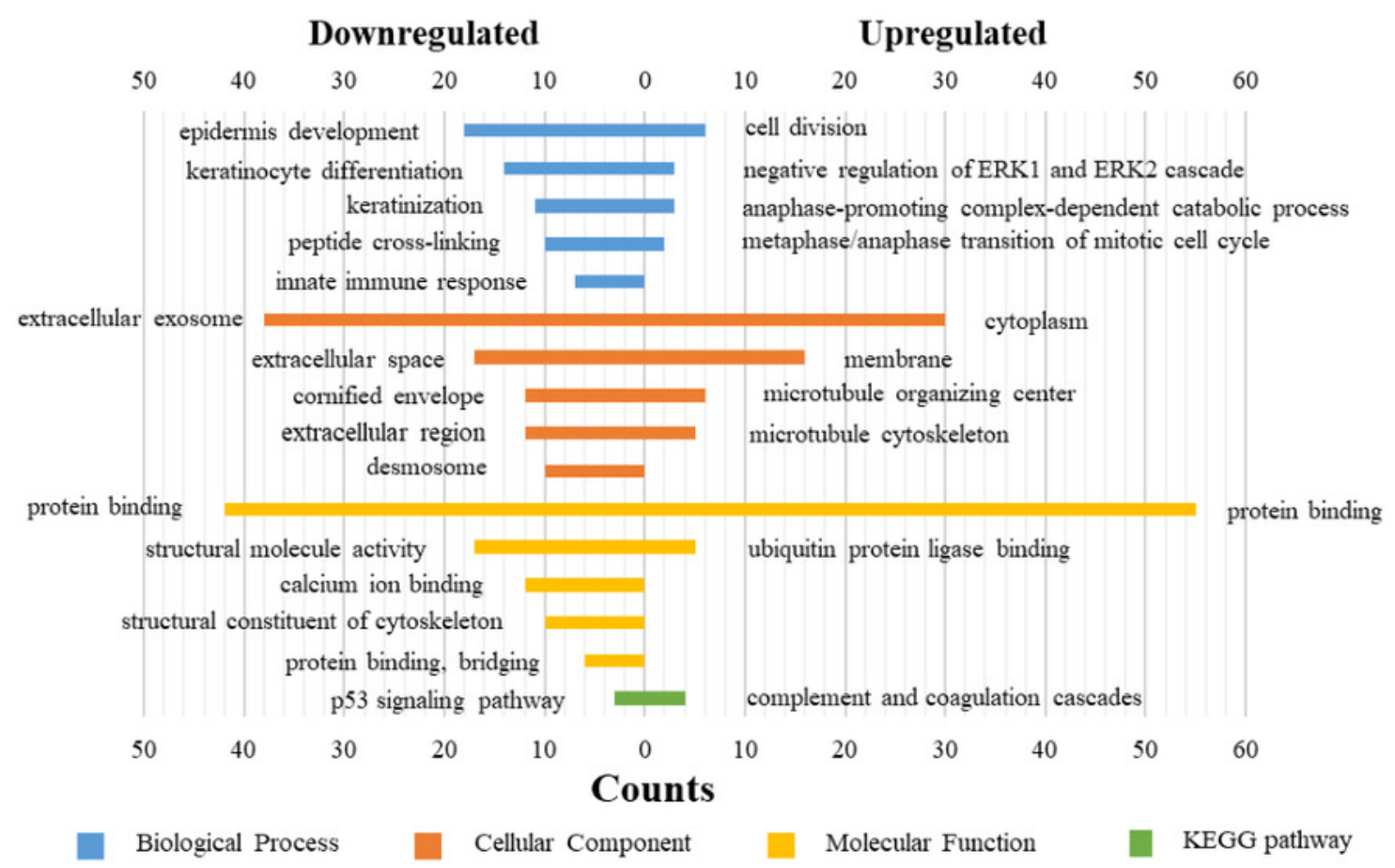


Figure 6

The PPI network of DEGs was constructed by using Cytoscape.

(A) PPI network of DEGs between PM and $\mathrm{N}$ of the three data sets. (B) PPI network of DEGs between MM and $\mathrm{N}$ of the three data sets. (C) PPI network of DEGs between MM and PM of the three data sets. 

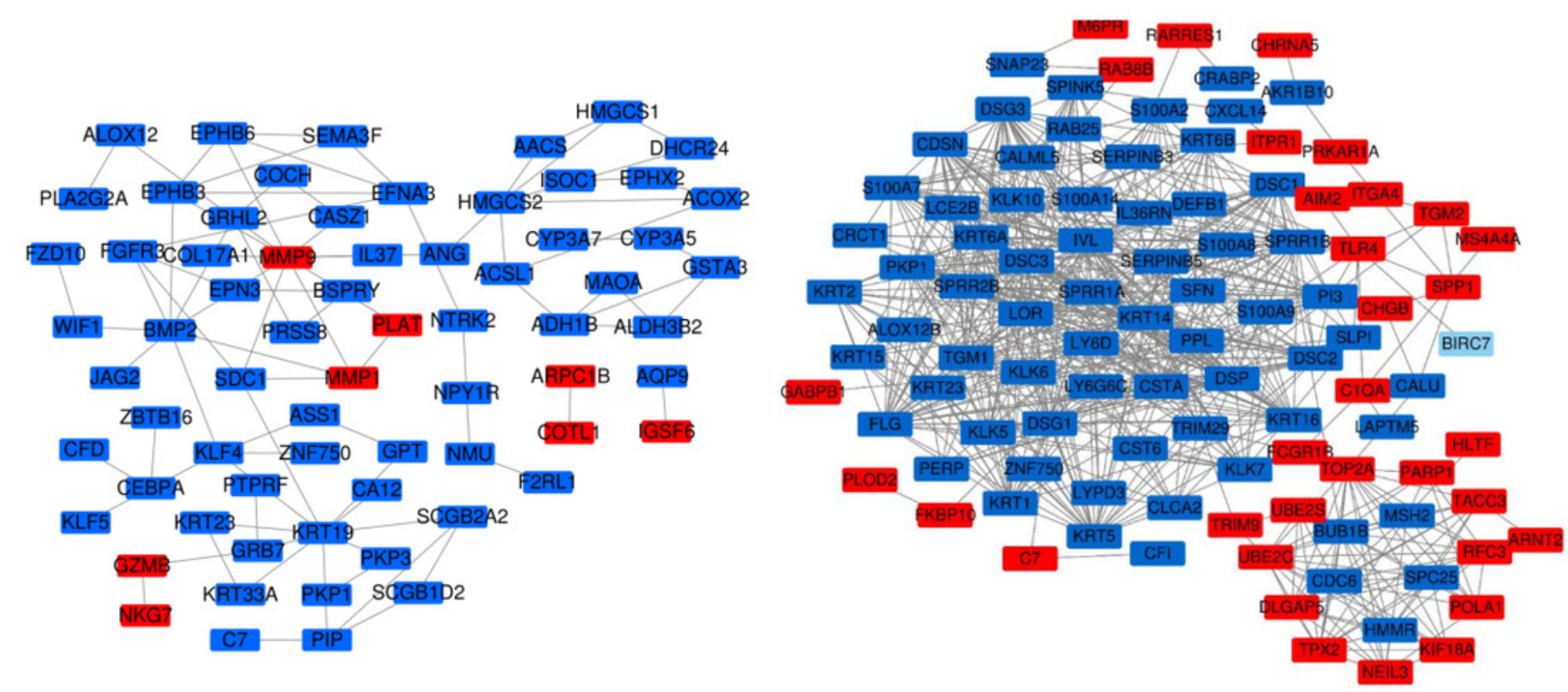

C

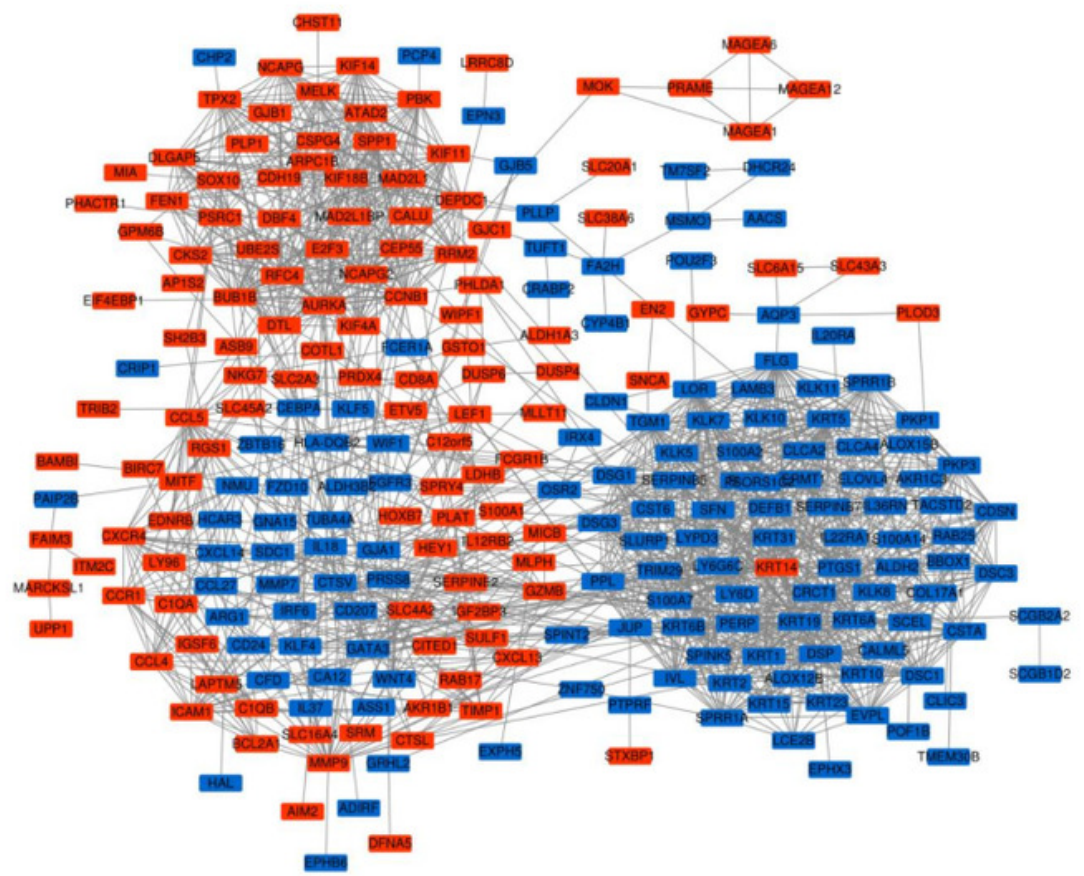




\section{Figure 7}

The most meaningful module from the PPI network.

Upregulated gene marker is light red, downregulated gene marker is light blue. (A) The most significant module of PM and N. (B) The most significant module of MM and N. (C) The most significant module of MM and PM.

A
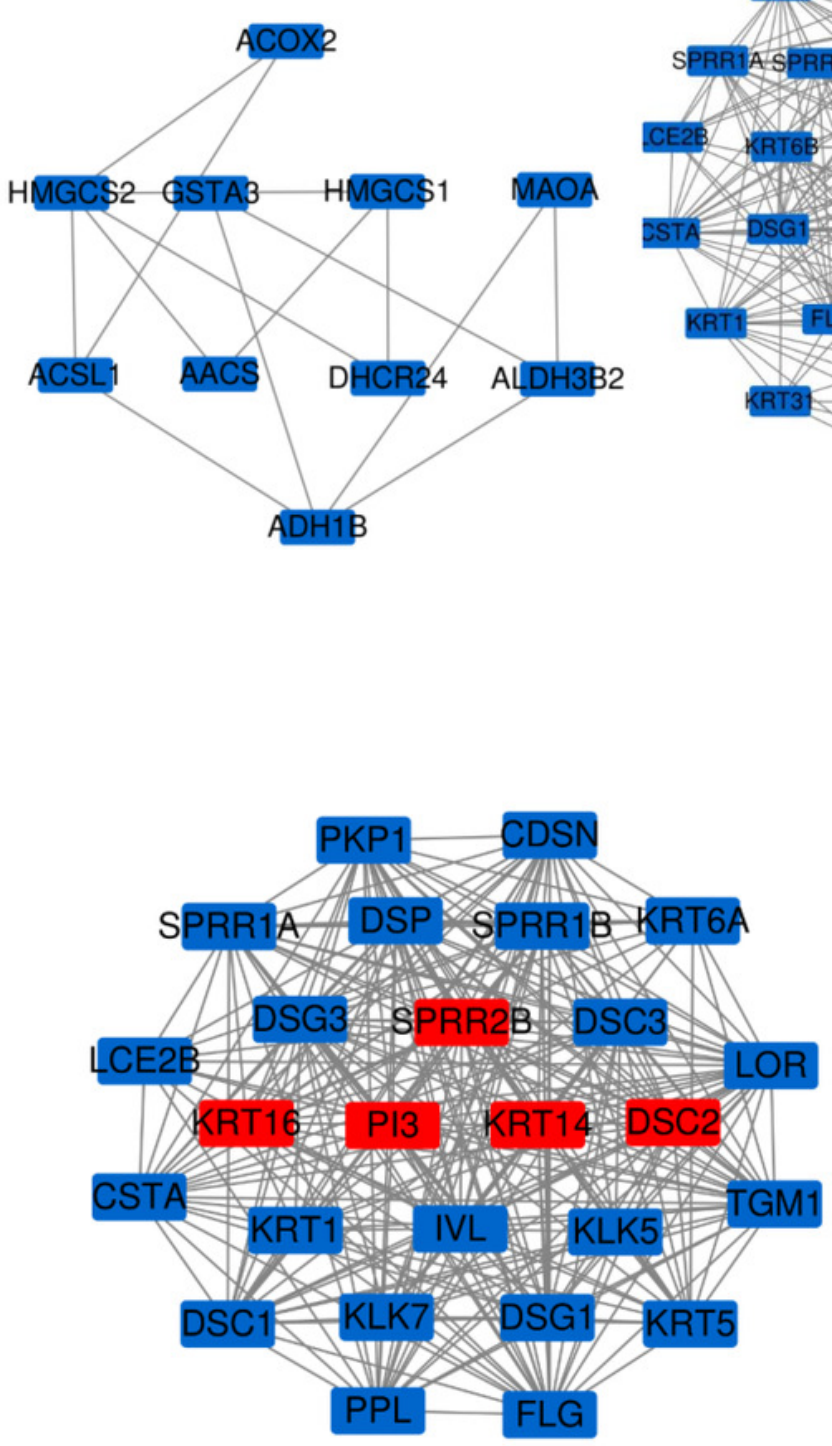

B

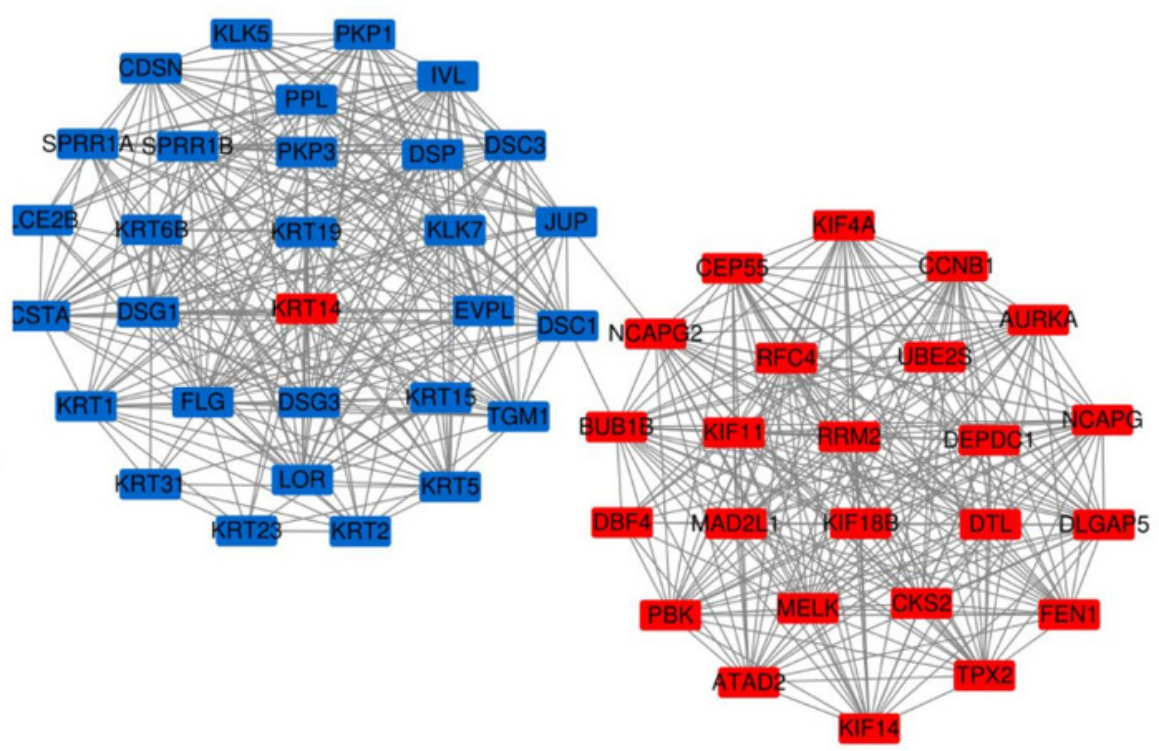

C

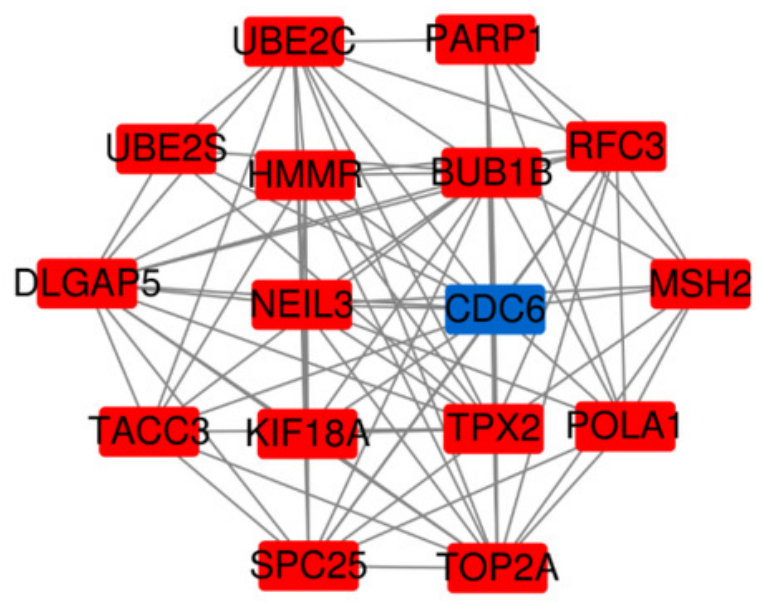




\section{Figure 8}

\section{GO functions and KEGG pathways enrichment analysis of the modular genes among normal skin, primary cutaneous melanoma and metastatic cutaneous melanoma.}

A

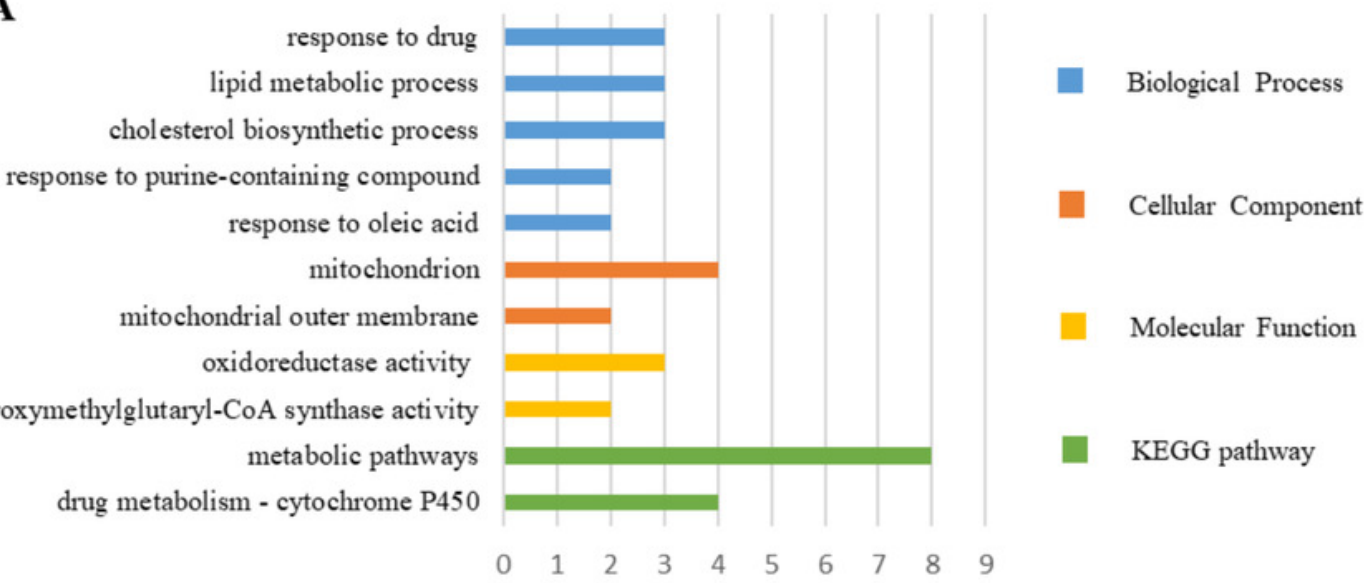

B

epidermis development cell division keratinocyte differentiation peptide cross-linking keratinization nucleus cytoplasm extracellular exosome desmosome intermediate filament protein binding structural molecule activity A TP binding structural constituent of cytoskeleton protein binding, bridging Cell cycle oocyte meiosis

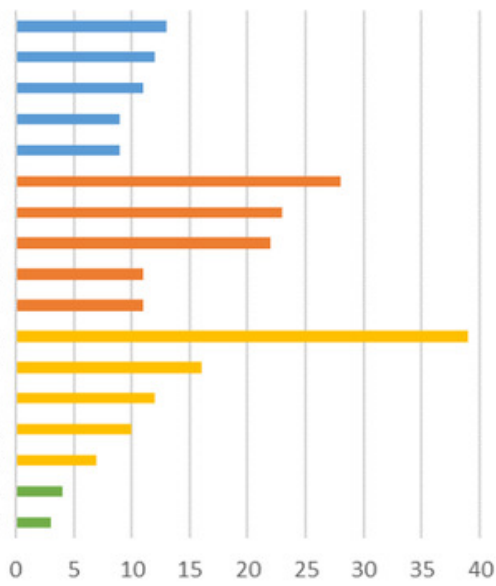

C keratinocyte differentiation epidermis devel opment keratinization peptide cross-linking cell division cytoplasm nucleus extracellular exosome cornified envelope desmosome protein binding structural molecule activity structural constituent of cytoskeleton protein binding, bridging calcium ion binding mismatch repair

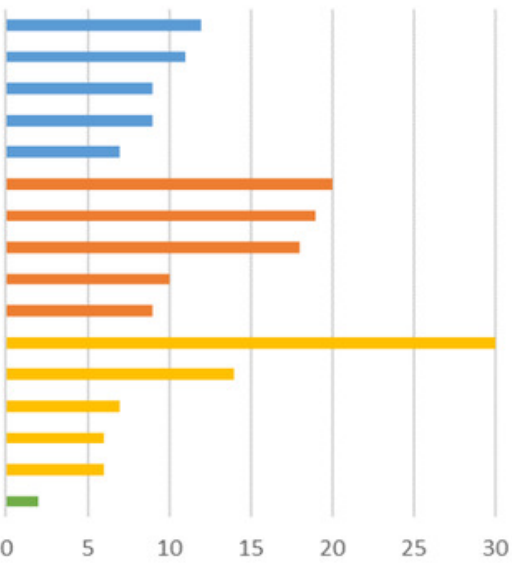


Figure 9

Box plot of gene expression values for hub genes in normal skin, primary and metastatic cutaneous melanoma samples.

(A) LOR (B) IVL (C) FLG (D) SPRR1B (E) DSG3 (F) KRT5 (G) CDSN (H) TGM1 (I) DSG1 (J) KRT16

(K) SPRR1A (L) PKP1 (M) KRT14 (N) DSC3 (O) DSP (P) CSTA (Q) S100A7 (R) DSC1, p<0.05 was considered statistically significant. ( $* p>0.05, * * p<0.05)$ 
A

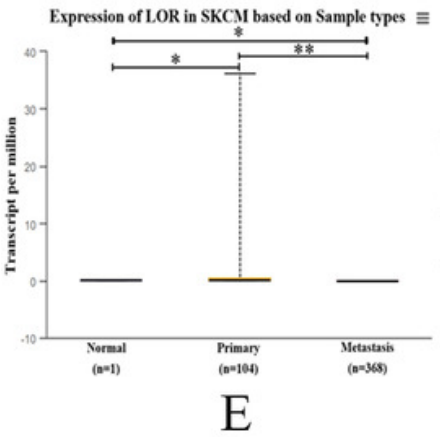

Expression of DSG 3 in SKCMI based on Sample types ミ
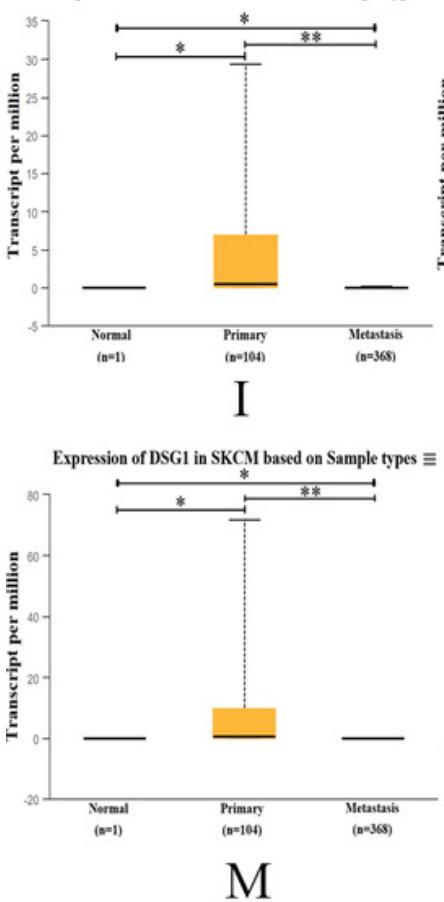

Expression of KRT14 in SKCMI based on Sample types $\equiv$

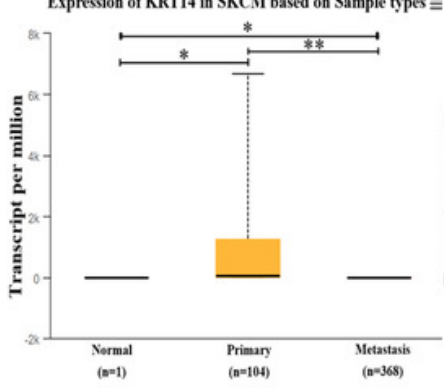

Q

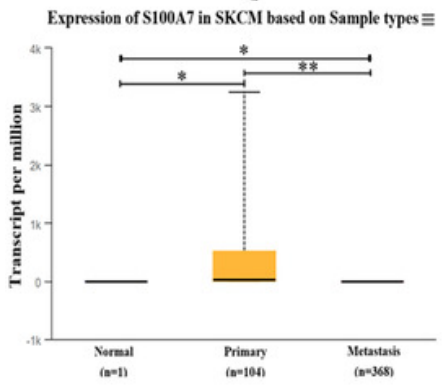

B

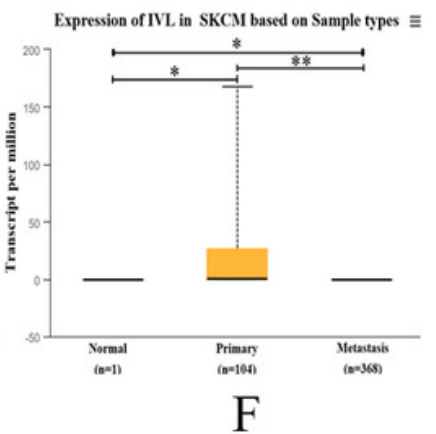

Expression of KRT5 in SKCM based on Sample types

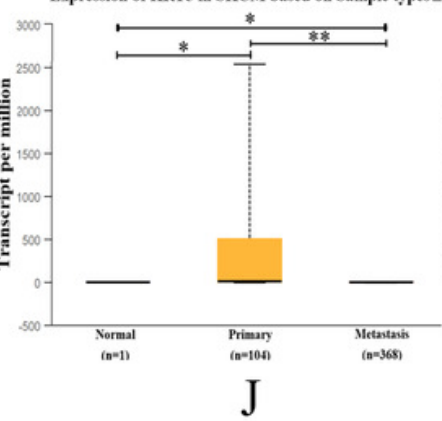

Expression of KRT16 in SKCM based on Sample types

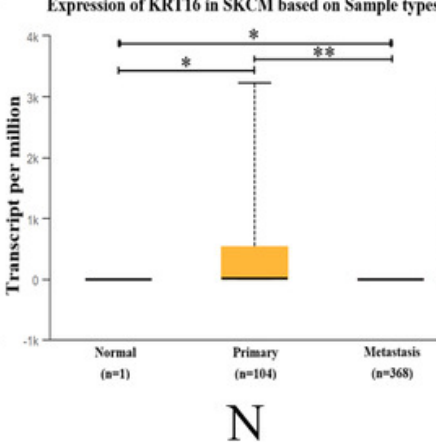

Expression of DSC 3 in SKCMI based on Sample types $\equiv$

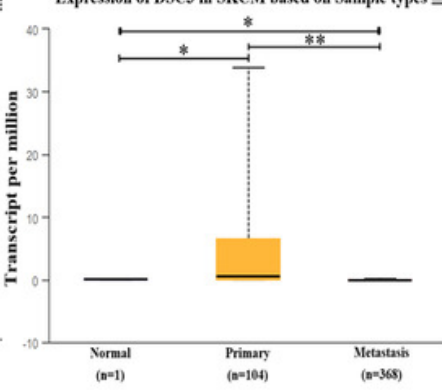

$\mathrm{R}$

Expression of DSC1 in SKCMI based on Sample types $\equiv$

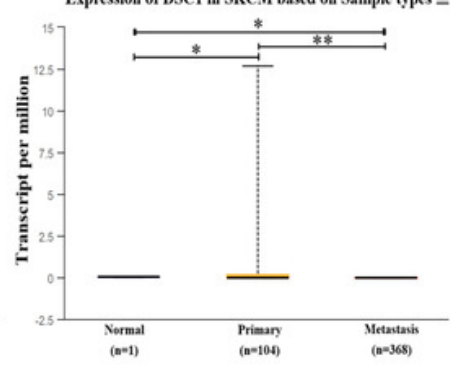

C
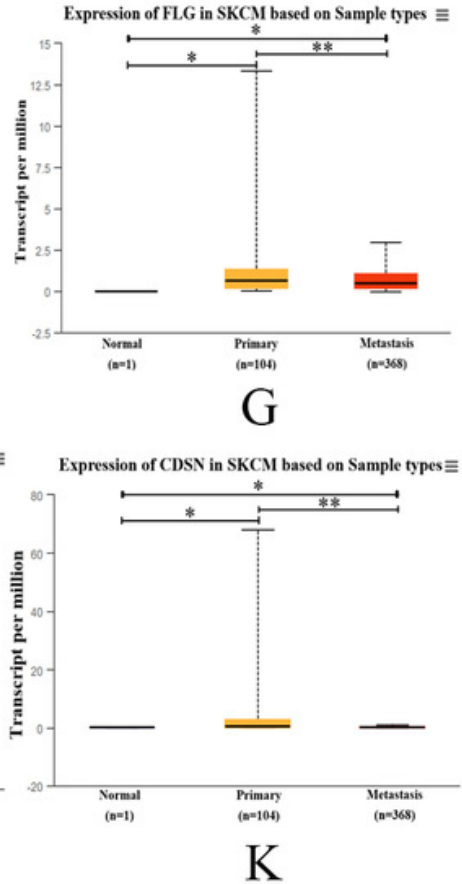

Expression of SPRR1A in SKCMI based on Sample types $\equiv$

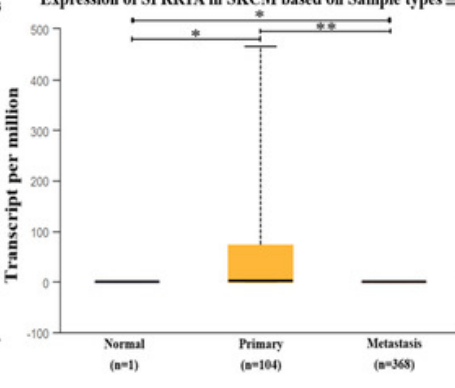

$\mathrm{O}$

Expression of DSP in SKCM based on Sample types $\equiv$

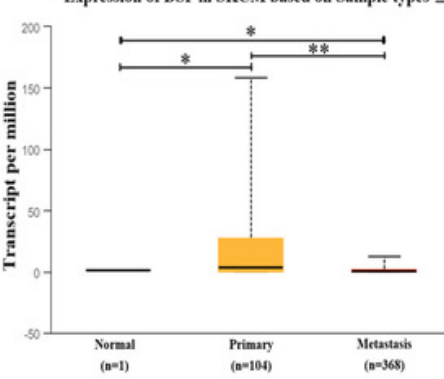

D

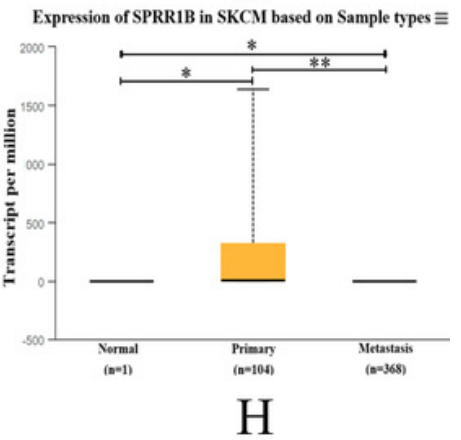

Expression of TGM1 in SKCM based on Sample types

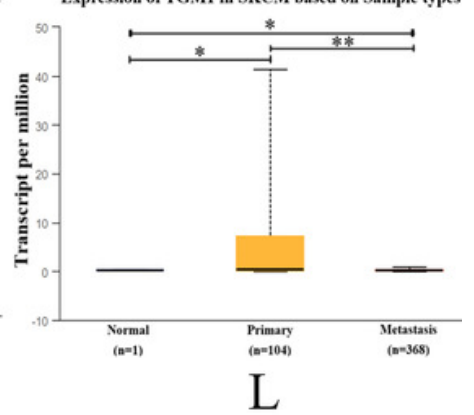

Expression of PKP1 in SKCM based on Sample types $\equiv$

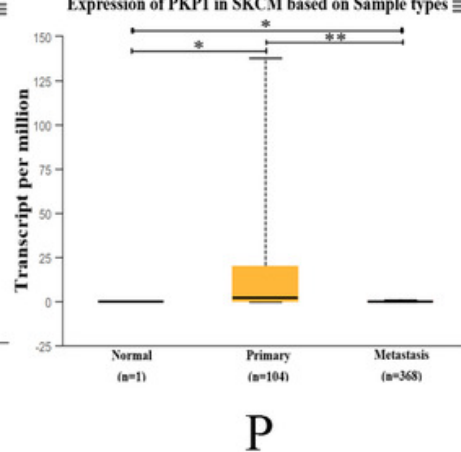

Expression of CSTA in SKCM based on Sanple

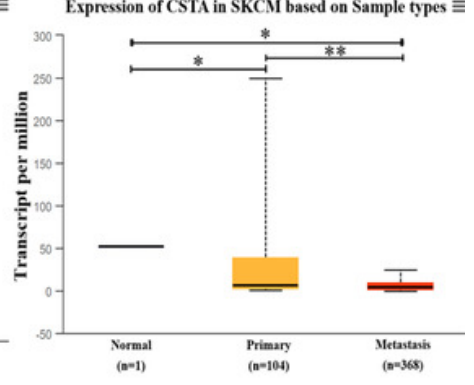


Figure 10

The overall survival curves of hub genes in TCGA database.

(A) IVL (B) FLG (C) SPRR1B (D) DSG3 (E) KRT5 (F) TGM1 (G) DSG1 (H) KRT16 (I) PKP1 (J)

KRT14 (K) DSC3 (L) DSP (M) CSTA (N) S100A7, p<0.05 was considered statistically significant. 
A

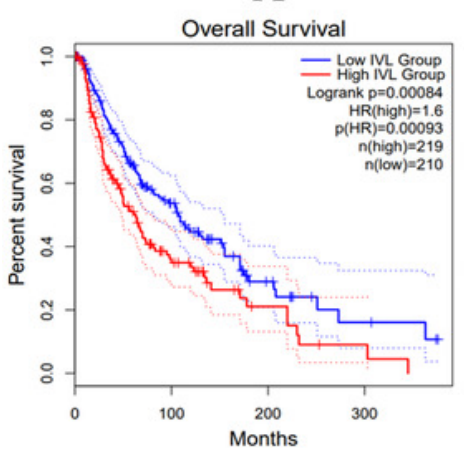

E

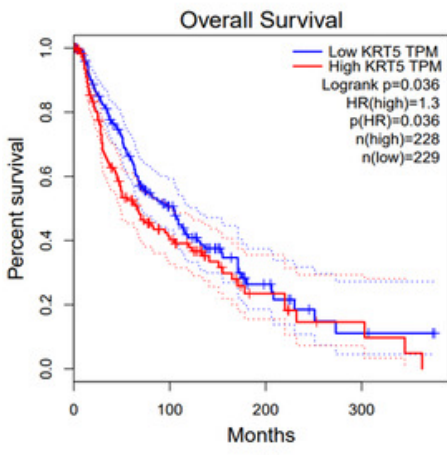

I

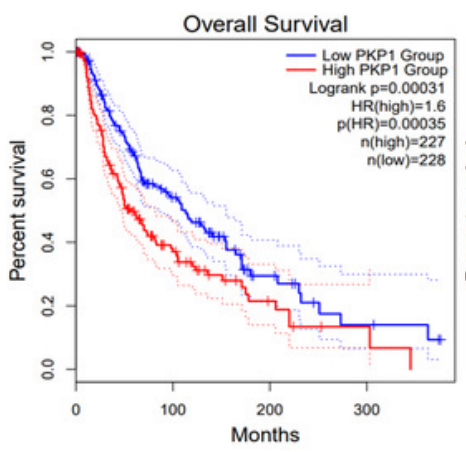

M

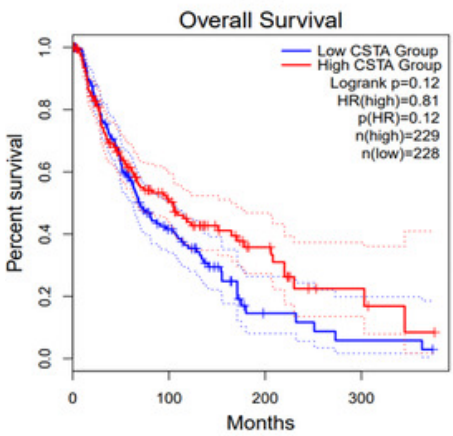

B

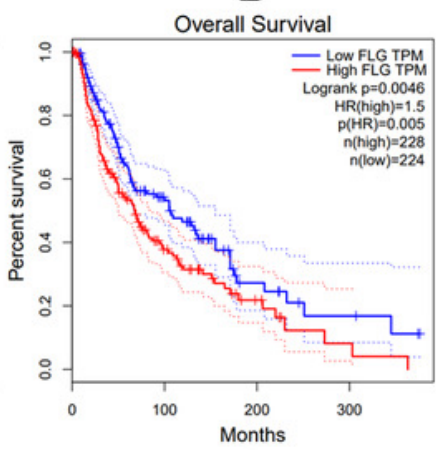

$\mathrm{F}$

Overall Survival

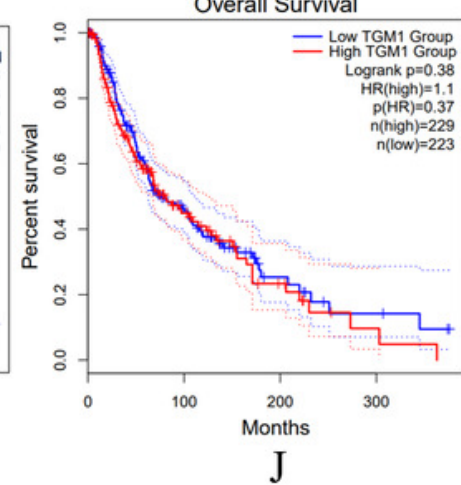

Overall Survival

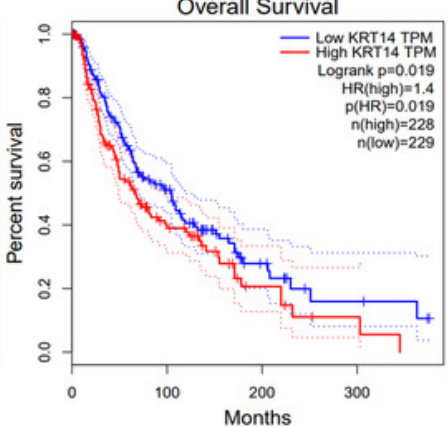

$\mathrm{N}$

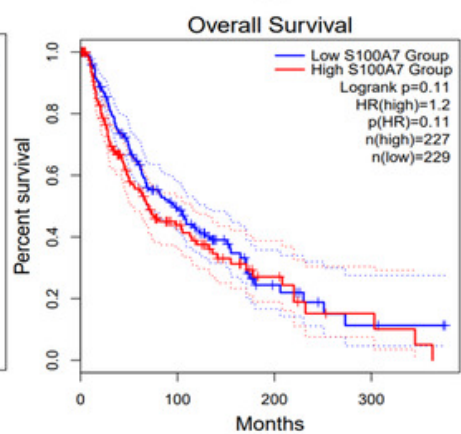

C

Overall Survival

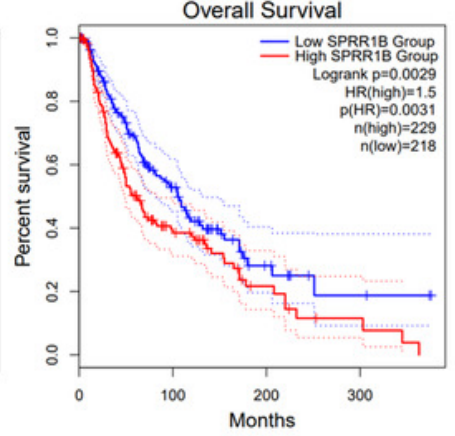

G

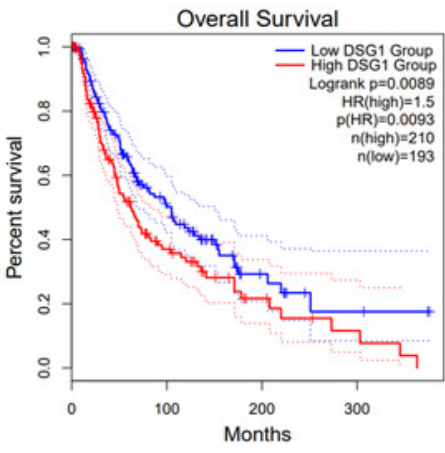

$\mathrm{K}$

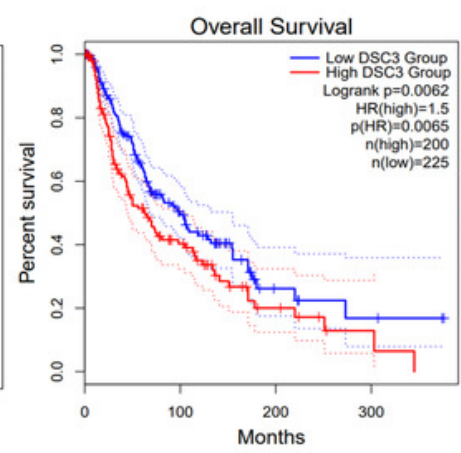

D

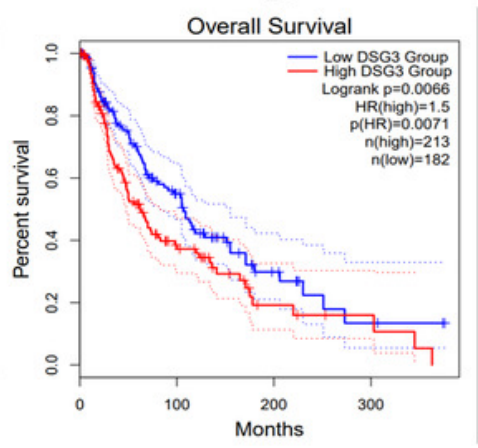

$\mathrm{H}$

Overall Survival

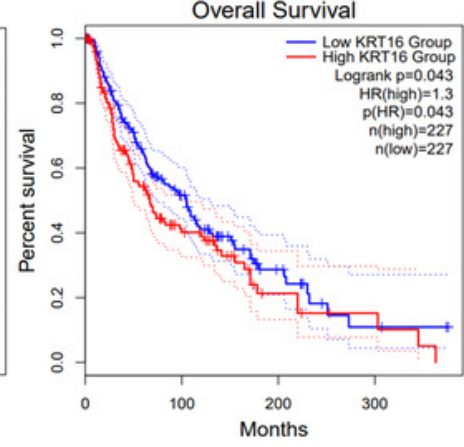

$\mathrm{L}$

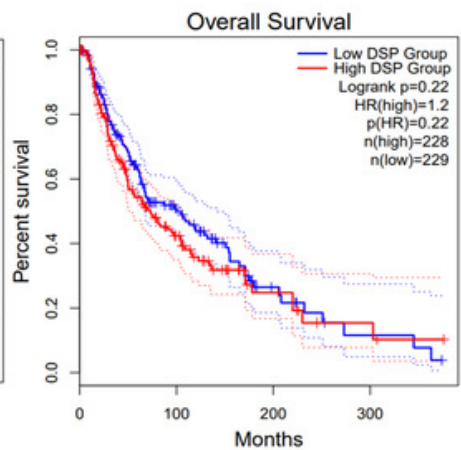

